\title{
Civilisations
}

Revue internationale d'anthropologie et de sciences

humaines

$53 \mid 2005$

Musiques "populaires"

\section{Les batá deux fois sacrés}

La construction de la tradition musicale et chorégraphique afro-cubaine

Kali Argyriadis

\section{(2) OpenEdition}

\section{Journals}

Édition électronique

URL : http://journals.openedition.org/civilisations/530

DOI : $10.4000 /$ civilisations. 530

ISSN : 2032-0442

Éditeur

Institut de sociologie de l'Université Libre de Bruxelles

\section{Édition imprimée}

Date de publication : 1 janvier 2006

Pagination : 45-74

ISBN : 2-87263-005-8

ISSN : 0009-8140

\section{Référence électronique}

Kali Argyriadis, «Les batá deux fois sacrés », Civilisations [En ligne], 53 | 2005, mis en ligne le 24 janvier 2009, consulté le 19 avril 2019. URL : http://journals.openedition.org/civilisations/530 ; DOI : 10.4000/ civilisations.530

(c) Tous droits réservés 


\title{
Les batá deux fois sacrés \\ La construction de la tradition musicale et chorégraphique afro-cubaine*
}

\author{
Kali ARGYRIADIS
}

Résumé : Au début du siècle, les musiques et les danses des descendants d'Africains à Cuba étaient considérées comme répugnantes et inaudibles, et leur pratique sévèrement réprimée. Aujourd'hui, les tambours batá et les danses d'orichas attirent un nombre croissant de visiteurs européens et américains dans l'île, en tant que paradigmes d'une supposée "pure tradition yoruba». Cet article tente d'analyser le rôle joué par les institutions (commerciales et nationales) et les intellectuels cubains dans le changement de statut de ce répertoire spécifique, ainsi que les interactions entre ces institutions et les pratiquants eux-mêmes, acteurs à part entière de cette évolution.

Mots-clés : batá, santería, Cuba, musique, construction de la tradition, religions afro-américaines.

Summary : At the beginning of the century, the musics and dances of Cubans of African descent were widely perceived as vulgar and unmusical, and they were consequently severely repressed. However, batá drums and orichas dances attract today a growing number of European and US visitors who see them as the paradigms of a supposedly "pure Yoruba tradition ". This article explores the role played by commercial and national institutions as well as Cuban intellectuals in the change of status of this specific repertoire. It also highlights the interaction between these institutions and the practitioners themselves, who are an integral part of this evolution.

Key words : batá, santeria, Cuba, construction of traditions, Afro-American religions.

* Je remercie Sara Le Menestrel, Stefania Capone, Silvina Testa et Véronique Boyer pour leur lecture attentive et leurs suggestions. 
T es pratiques religieuses d'origine africaine et leurs expressions artistiques ont Llongtemps été méprisées à Cuba. Au sortir de l'indépendance, au début du siècle, la musique et les danses « de nègres » étaient fortement réprouvées et réprimées. Pourtant aujourd'hui, alors que depuis une dizaine d'années l'île s'est ouverte au tourisme, elles sont devenues l'un des facteurs d'attraction majeure des visiteurs de ce pays. Enthousiasmés par une production et un enseignement de qualité adaptés à leurs attentes, certains poussent leur passion jusqu'à l'engagement religieux (Argyriadis, 2001-2002). D'autres les découvrent tout simplement à travers les voyages organisés qui incluent à leur programme nombre « d'activités culturelles » basées sur un répertoire parfois encore appelé « folklorique », et plus souvent «traditionnel afro-cubain ». A La Havane, aux côtés des indétronables Guantanamera, Hasta siempre et autres Chan chan, pas un musée, marché artisanal, lieu de spectacle ou même colloque international qui ne soit saturé de rumbas, tambours batá et chants et danses d'orichas.

Les cérémonies religieuses qui ont lieu quotidiennement dans la capitale sont pourtant loin de se restreindre à l'emploi de ce type de tambours, de chants et de gestuelles. De même, les orichas ne sont pas les seules entités à être invoquées : les saints et les vierges, ainsi que différentes sortes d'esprits de défunts sont également mobilisés de façon complémentaire (Argyriadis, 1999). Cependant, la santería et son corollaire, la divination par ifá occupe une place hégémonique en tant que paradigme d'une prestigieuse tradition africaine «yoruba ", tandis que son répertoire musical et chorégraphique est de loin le plus plébiscité à la fois par les artistes, leur public et les institutions nationales. Les tambours batá et les danses d'orichas sont devenus l'incarnation d'une culture légitimée, valorisante, symboles parmi d'autres de l'identité nationale cubaine. Dans le contexte de transnationalisation de la santería (Argyriadis, Capone, 2004) ils en viennent même à être présentés comme porteurs d'une traditionnalité yoruba plus pure et plus noble qu'en Afrique.

Le répertoire «afro-cubain » a donc changé radicalement de statut en moins d'un siècle. En devenant musique savante, musique d'initiés dans les deux sens du terme, il a aussi évolué en mettant l'accent sur un nombre restreint de ses variantes. L'émotion esthétique qu'il suscite de nos jours auprès d'un public international est le fruit d'un processus d'interactions vieux de plus d'un siècle, qu'il est essentiel d'analyser pour comprendre les enjeux contemporains de la pratique religieuse santera à Cuba et hors de l'île. Ces échanges sous-tendent en outre un rapport à l'Afrique qui n'a jamais cessé d'être ambivalent dans la notion de cubanité, et dont la profondeur historique doit être revisitée.

\section{Afrocubanisme et esthétique du rythme}

Lorsqu'en 1898 l'île de Cuba acquit son indépendance, la question de l'intégration des descendants d'esclaves dans le concept d'identité nationale ${ }^{1}$ n'allait pas de soi, et ce malgré leur participation massive aux débats et aux luttes abolitionnistes et anti-colonialistes

1. La cubanité était censée transcender les phénotypes. Des mouvements comme le soulèvement du Parti des Indépendants de Couleur, anciens combattants de la guerre d'indépendance qui dénoncèrent l'absence de personnes « de couleur » au gouvernement, furent sévèrement réprimés (1912, environ 3000 morts). 
de la fin du $19^{\text {e }}$ siècle. La nouvelle république se préoccupait de « désafricaniser »sa population et amorça très vite une politique de "blanchissement », en subventionnant l'immigration espagnole. Les premières études sur les pratiques des descendants d'esclaves furent le fait de fonctionnaires de police, de juristes et de médecins légistes. Comme au Brésil (Capone, 1999 : 207) il s'agissait de se donner les moyens d'éradiquer des activités considérées comme délictueuses et honteuses pour la jeune nation, comme la «sorcellerie » ou la prolifération de sociétés secrètes masculines d'origine africaine dites de ñáñigos. Mentionnés dès 1882 (Trujillo y Monagas), ces derniers furent l'objet de persécutions policières répétées. On leur confisquait leurs objets, instruments et costumes rituels, pour les placer au Museo de Ultramar de Madrid. C'est là qu'un jeune Cubain étudiant en droit, Fernando Ortiz, les vit en 1901. A l'instar du public espagnol de l'époque, il trouva le sujet fascinant (Ortiz, 1939 : 86).

La même année à La Havane, un décret municipal avait interdit l'usage de tambours d'origine africaine. Les journaux de l'époque étaient remplis d'anecdotes illustrant la nécessité de la répression anti-sorciers et de la confiscation de leurs fétiches, tambours et ingrédients suspects. Les sorciers africains étaient soupçonnés (voire accusés et condamnés) de sacrifices humains et de charlatanisme. On leur reprochait aussi de violer les sépultures et de se livrer à des crises d'épilepsie incontrôlées. Plusieurs pamphlets traitèrent le problème en terme de pathologie sociale (Valero Cossio, 1904; Roche Monteagudo, 1908; Castellanos Gonzalez, 1916), mais c'est celui de F. Ortiz qui se distingua (Los negros brujos, 1906), car il introduisait le terme « afro-cubain » pour désigner spécifiquement les quelques milliers de natifs africains âgés vivant à Cuba (et non les millions de descendants d'Africains nés dans l'île, comme l'usage le fit par la suite), et s'appuyait sur des sources africanistes pour montrer qu'il s'agissait de survivances religieuses de divers peuples subsahariens, dont il essayait de constituer la liste.

Comme ses contemporains, F. Ortiz jugeait ces pratiques attardées et amorales et appelées à disparaître. Il considérait avec bien plus de sévérité celles des Cubains qui les perpétuaient en faisant consciemment œuvre de charlatanisme et de parasitisme, et il distinguait soigneusement ces derniers des « Noirs évolués », c'est-à-dire de la bourgeoisie et des intellectuels « de couleur» de l'époque qui étaient les premiers à manifester leur dégoût de la sorcellerie. Il prôna la « désafricanisation » (Ortiz, 1995 : 150) par le biais de l'éducation et de l'accès aux soins pour tous, et demanda à ce que les objets confisqués ne soient pas détruits mais donnés au Musée d'anthropologie de l'Université nationale « dans l'intérêt de la science» (idem : 196). Son opinion sur les pratiques artistiques afro-cubaines était à ce moment-là très méprisante : dans Los negros brujos, les danses sont «lascives de façon dégoûtante», sauvages et antisociales (idem : 46, 183); les chants « se réduisent à des répétitions rythmiques d'une insistance désespérante pour les oreilles cultivées » (idem : 47). F. Ortiz s'étonne d'ailleurs de la pénétration de ces cultes «dépourvus de forme artistique civilisée» dans les couches sociales élevées (idem : 138), et préconise des mesures plus répressives encore envers les « danses africaines », dangereux facteur d'attraction de nouveaux adeptes, car dissimulant souvent des fêtes religieuses sous couvert de divertissement (idem : 200).

Dans la lignée de ce jugement partagé par les pouvoirs publics, les groupes carnavalesques (comparsas) furent interdits en 1913. Patriote engagé, athée convaincu, F. Ortiz se consacra ensuite à l'étude du spiritisme kardéciste et de l'archéologie indocubaine, et poursuivit dans un premier temps des recherches plus historiques sur les afrocubains (rébellions, esclavage, associations et pratiques festives du $19^{\mathrm{e}}$ siècle...). 
Dans les années vingt, l'île connut une grave crise économique due à la baisse du cours du sucre. Les grandes compagnies sucrières répondirent aux mouvements sociaux par l'immigration "sous contrat» de plusieurs centaines de milliers de Jamaïcains et d'Haïtiens. Alors que les chasses aux sorciers reprenaient de plus belle, plusieurs voix s'élevèrent contre la présence nord-américaine à $\mathrm{Cuba}^{2}$ et proposèrent en réaction une définition de la cubanité qui admettait une part d'influence africaine, au moins sur les religions et les arts. Des journalistes, des écrivains et des hommes politiques de toutes couleurs de peau érigèrent le danzón et surtout le son en symboles nationaux. Selon A. Carpentier, « Seuls les noirs [...] conservaient jalousement un caractère et une tradition antillaise. Le bongó, antidote de Wall Street ! » (cité par De la Fuente, 2001 : 243).

En 1923 F. Ortiz s'unit à la campagne de la Junte de rénovation nationale civique, qui évolua en rébellion rapidement avortée. Ces organisations inspirèrent un groupe (dit Minoriste) de jeunes artistes et intellectuels qui se réunirent autour de conférences, d'expositions et de concerts, et découvrirent les nouveaux mouvements artistiques européens, notamment le primitivisme et l'Art Nègre. Ils remarquèrent alors «qu'il y avait à Regla, de l'autre côté de la baie, des rythmes aussi complexes et intéressants que ceux que Stravinski avait créé pour évoquer les jeux primitifs de la Russie païenne » (Carpentier, 1985 : 286). Or, si le folklore européen était considéré comme agonisant, les expressions populaires créoles cubaines étaient elles « en continuel processus de création » et source d'orgueil (idem). Le mouvement, appelé afrocubaniste, prit F. Ortiz comme porte-étendard. Ce dernier, «malgré son âge, se mêla fraternellement à la jeunesse. On lut ses livres. On exalta les valeurs folkloriques. Subitement, le Noir devint le centre de l'attention de tous les regards. Du fait même que l'on faisait enrager les intellectuels de la vieille école, on allait avec onction aux juramentos ñánigos et l'on portait aux nues la danse des diablitos » (idem : 267-268). F. Ortiz co-fonda en 1923 la Société de Folklore Cubain, puis la revue Archivos del folklore cubano, et entreprit ses recherches sur la musique, les danses et le théâtre des « Noirs ».

L'afrocubanisme se développa dans le contexte extrêmement propice de l'engouement pour le son. Privés de carnaval, interdits dans les fêtes profanes et religieuses ${ }^{3}$, les tambours et les rythmes populaires n'eurent longtemps droit de cité officiel que dans les théâtres bouffes, comme l'Alhambra, où se produisaient tous les grands musiciens, danseurs et chanteurs du début du siècle. C'est là que dès 1906, des maisons de disques américaines (Edison, puis Victor, Columbia...) vinrent enregistrer sur cylindres de métal, pour leurs " séries ethniques », des rumbitas guaracheadas accompagnées à la guitare ou au piano (Reyes, $2000: 34$ ). De ce fait, le terme rumba $a^{4}$ devint très tôt à la mode aux Etats-Unis,

2. L'Amendement Platt, signé en 1902, donnait le droit d'intervention aux troupes US (qui en usèrent notamment en 1906, 1912 et 1917). Un Traité de réciprocité commerciale stipulait que les Etats-Unis achèteraient tout le sucre cru, en échange de produits manufacturés.

3. Ce qui ne veut pas dire que les gens cessèrent de jouer; les tambours risquant d'être confisqués, ils les remplaçaient souvent par des tabourets et des caisses vides, facilement escamotables en cas d'irruption policière.

4. Qui désigne un genre musical et chorégraphique distinct du son : la base rythmique (clave) est différente, les danseurs ne sont pas enlacés, le contexte d'exécution contemporain courant reste, outre les spectacles folkloriques, celui des cours d'immeubles populaires, des fêtes familiales et religieuses. A Cuba on distingue donc la rumba de la rumba de salón. 
et c'est pourquoi il fut utilisé plus tard dans les années vingt pour désigner le son, lorsque la musique cubaine pénètra massivement ce pays.

Le son envahit la capitale dans les années vingt en même temps que les immigrants de la région orientale de l'île dont il était issu. Les afrocubanistes le découvrirent avec une «stupeur émerveillée » (Carpentier, 1985 : 216) et virent en lui la première vraie création cubaine - du moins sous sa forme première, car son évolution jazzistique fut au contraire très critiquée ${ }^{5}$. Ses instruments étaient pourtant de facture très rustique ${ }^{6}$, chacun servant à produire des timbres et des phrases rythmiques entrecroisées. Mais les « oreilles cultivées » avaient désormais de nouvelles références : «La grande révolution opérée dans les idées reçues par la batterie du son consista à nous donner le sens de la polyrythmie soumise à une unité de temps. (...) Le son cubain - tel qu'il se présenta à nous dans sa forme pure, en 1920 - fait penser à un état rudimentaire des Noces de Stravinski » (Idem :

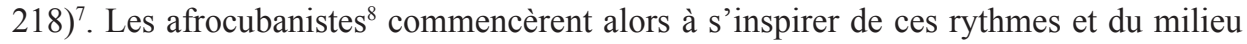
dont ils étaient issus, faisant scandale parmi les partisans de l'hégémonie de la musique paysanne (guajira) d'origine espagnole.

De 1925 à 1933, sous la dictature de Machado, de nombreux afrocubanistes s'exilèrent en France et se lièrent avec les surréalistes. Ce fut par exemple le cas d'A. Carpentier, que Robert Desnos emmena avec lui à sa sortie de prison en 1927. A Paris, l'engouement pour les danses exotiques battait son plein, et les surréalistes se pressaient au Bal nègre de la rue Blomet. Au-delà de toute l'ambiguïté de ce phénomène, qui mêla souvent gêne et fascination, ces bals furent des lieux propices aux rencontres entre intellectuels Antillais, Africains, Noirs américains, et ils contribuèrent à l'émergence du courant de la négritude (Decoret, 1998) ${ }^{9}$. Ces rencontres, cette aura de danses considérées à Cuba comme répugnantes, inspirèrent très certainement les artistes cubains, d'autant que dès 1931 la mode de la « rumba » et de la conga arriva à son tour à Paris via les Etats-Unis.

5. La musique américaine et surtout le jazz influencèrent la musique de l'île dès les années vingt. Les sextets de son remplacèrent la marímbula par la contrebasse, introduisirent l'usage de la trompette et devinrent des septets. Comme le souligne L. Acosta (2001 : 37), de riches échanges musicaux ont nourri les créations musicales des deux pays. Les exemples les plus marquants dans les années quarante sont ceux à Cuba des jazz bands de Benny Moré et de Damaso Pérez Prado (à l'origine du mambo), et aux USA de l'orchestre Machito \& his AfroCubans, dirigé par Mario Bauzá, et de la collaboration entre le Cubain Chano Pozo et Dizzy Gillespie (1948), qui contribuèrent, de par leurs innovations rythmiques, à la création du Latin Jazz.

6. Jarre en terre cuite (botija) dans laquelle on soufflait, lamelles de métal montées sur une caisse de résonance (marímbula), bâtons entrechoqués (claves), maracas, petit tambour (bongó), calebasse striée frottée (güiro), petite guitare à trois paires de cordes (tres).

7. Rappelons que l'orchestre des Noces (1923) était uniquement composé de voix, de quatre pianos et de percussions. On pourrait également souligner l'impact de la chorégraphie « ensauvagée » de Nijinski pour le Sacre du printemps, qui fit scandale en 1913.

8. Amadeo Roldán composa par exemple en 1925 son Ouverture sur des thèmes cubains. A. Carpentier écrivit en prison son livre Ekue Yamba-o (1927), histoire d'un noir guajiro de famille santera qui devient ñáñigo à La Havane et connaît un destin tragique. L'œuvre fut transposée en opéra par le compositeur Alejandro García Caturla en 1931. En 1930, Nicolás Guillén composa son recueil de poèmes engagés Motivos de son, mis en musique par A. Roldán en 1934.

9. C'est dans cette ville, en 1929, qu'A. García Caturla joua pour la première fois, avec le soutien des surréalistes, son œuvre Bembé, du nom de la fête où l'on joue des tambours bembé en l'honneur des orichas. Les Petits contes nègres de Lydia Cabrera furent publiés pour la première fois en français à Paris, en 1936, et L. Cabrera traduisit le Cahier d'un retour au pays natal d'A. Césaire en espagnol en 1943). 
Mais ce nouveau regard, esthétiquement valorisant, n'impliquait pas la remise en cause de certaines hiérarchies. A propos de la composition Rythmiques, d'A. Roldán (1930), où auraient été pour la première fois notés avec exactitude les rythmes de percussions afrocubaines, A. Carpentier s'extasiait sur la capacité de "l'artiste cultivé » à faire œuvre de recréation, à travailler l'esprit d'un folklore, là où le musicien noir n'aurait fait que frapper «instinctivement» ses tambours (Carpentier, 1985 : 276). Il s'agissait donc bien de puiser des sources d'inspiration, mais en aucun cas de légitimer des pratiques religieuses jugées criminelles et violentes, qu'A. Carpentier associait comme F. Ortiz au manque d'instruction, coupable du fait que tant de Noirs « végètent dans la pègre avec un tambour sur le ventre » (idem : 251).

Il convient toutefois de nuancer le constat de cette restriction au champ esthétique en rappelant que d'autres intellectuels de l'époque, s'auto-affirmant « de couleur», entendaient faire valoir un point de vue plus radical. Réunis autour du Club Atenas, nouant des liens avec les intellectuels du mouvement artistique new-yorkais contemporain Harlem Renaissance (qui influencèrent aussi la poésie de N. Guillén), ils s'exprimèrent dans les années trente à travers une série d'articles de G. Urrutia dans le Diario de la Marina, intitulés « idéaux d'une race », engagés contre le racisme et la ségrégation à Cuba $^{10}$ comme aux USA (Castañeda, 2001 : 235-236). Ils refusaient la création d'un mouvement purement afro-cubain (le terme étant entre-temps devenu synonyme de descendant d'Africain) et la ghettoïsation qui en aurait résulté, mais soulignaient « l'apport de la culture afro-cubaine à notre civilisation nationale ». Il ne s'agissait donc plus ici seulement d'esthétique : G. Urrutia s'éleva d'ailleurs contre la spectacularisation des art afro-cubains, ironisant au sujet des peuples européens qui, « pour se refaire depuis la moelle psychologique, ont besoin d'une très forte injection de sauvagerie cultivée » (Urrutia, 1935).

Après la dictature de Machado et l'abrogation de l'amendement Platt, l'afrocubanisme gagna peu à peu les institutions. Mais surtout, il prit un nouveau tournant, en orientant sa démarche vers la détection de survivances africaines à Cuba, et la valorisation extrême des pratiques attribuées aux descendants des Yoruba. Le répertoire musical et chorégraphique des cultes commença à être analysé à la loupe, puis hiérarchisé, à partir de critères d'authenticité traditionnelle toujours étroitement liés aux critères esthétiques, aussi bien des universitaires que de leurs informateurs.

\section{La consécration des batá lucumí}

$\mathrm{Au}$ contraire des «Congos», dont les pratiques, qualifiées péjorativement de syncrétiques, sont devenues à partir de cette époque le paradigme d'une Afrique honteuse, laide, sale, sauvage et sorcière, les "Yorubas " sont présentés aujourd'hui encore comme les héritiers d'une civilisation savante, belle, prestigieuse et «traditionnelle » (Argyriadis, 2000). A l'instar de son précurseur brésilien R. Nina Rodrigues, F. Ortiz a été influencé par les stéréotypes raciaux qui avaient cours en son temps, les « Soudanais »

10. L'époque n'était pas favorable, dans les faits, à ceux qui étaient toujours désignés comme sorciers. La répression et les confiscations d'objets rituels se poursuivaient, ainsi que les pamphlets journalistiques, tandis que se créait 1934 le mouvement ABC, proche du Ku-Klux-Klan. 
étant considérés comme plus beaux et plus susceptibles d'être civilisés que les "Cafres » (Capone, 2000 : 6). Grand lecteur des travaux de A. Hovelacque, M. Delafosse et C. Lombroso, l'idée d'une supériorité de la culture yoruba lui vint également des textes du missionnaire T. J. Bowen, de l'abbé P. Bouche, du révérend S. A. Crowther et du colonel A. B. Ellis, acteurs du mouvement de renaissance culturelle de Lagos qui visait, dans la seconde moitié du $19^{\mathrm{e}}$ siècle, à jeter les bases d'une nation dite yoruba, munie d'une langue standard et d'une religion commune, élevée au rang de dignité de la religion chrétienne (Matory, 2001 : 175). F. Ortiz exulta donc lorsqu'en 1916 (dans Los negros esclavos) il fit enfin le lien entre ces mythiques Yoruba et les esclaves appelés à Cuba lucumí (1995: 27, note de l'éditeur).

En 1939, un autre chercheur, R. Lachateñeré, s'appliqua à distinguer soigneusement les pratiques palo-mayombe (congo) des lucumí. Il considérait le terme de sorcellerie impropre pour qualifier ces dernières, et il suggérait de lui préférer le terme utilisé par les intéressés, à savoir santería. F. Ortiz acquiesça et se réjouit de l'existence d'un vocabulaire moins péjoratif. Dans ses textes ultérieurs, R. Lachateñeré distingua soigneusement les «Bantous » des «Yoruba », et développa l'idée d'une subordination progressive des autres cultes africains à la religion yoruba.

Ces théories ont sans doute été également inspirées aux universitaires cubains par certains de leurs informateurs, soucieux d'échapper aux persécutions et de se démarquer du stigmate de sorciers qui pesait sur eux. F. Ortiz précise que vers 1906, le terme était en fait utilisé par les babalaos pour désigner les paleros (1939 : 88). R. Lachateñeré affirme quant à lui avoir été guidé par « les discussions théologiques et dogmatiques engagées par les prêtres de l'un et l'autre groupe ». Il remarque qu'il faut mettre ces discriminations sur le compte des raisons économiques et des jalousies professionnelles, preuve en est que certains paleros accusent à leur tour les santeros d'être des sorciers. Mais il conclut tout de même : «Cependant, à notre avis il faut accepter l'idée d'un plus grand contenu dogmatique dans les cultes lucumí » (1940 : 6). Al'exception de L. Cabrera, les chercheurs ne prirent pas en compte le cumul des pratiques, extrêmement courant dès cette époque, et tinrent ces accusations pour l'expression d'un antagonisme entre groupes distincts.

Dès lors qu'ils constatèrent l'engouement pour la santería, les religieux mirent en avant cette modalité de culte. Nicolás V. Angarica, santero célèbre, auteur entre autres d'un manuel religieux intitulé El lucumí al alcance de todos écrit vers 1957, omit ainsi prudemment de préciser qu'il pratiquait aussi le palo et introduisit ainsi son propos : « Cette religion ressemble beaucoup à la catholique, elle n'a rien de répugnant, comme beaucoup par convenance on voulu la faire paraître, au moyen de la presse et des autres moyens de divulgation et publicité » (Angarica, 1990 : 9). Il s'employa ensuite à convaincre le lecteur de la valeur culturelle de sa religion, en proposant notamment un dictionnaire espagnol-lucumí directement inspiré du dictionnaire anglais-yoruba de la Oxford University Press (León, 1971 : 143), des passages d'ouvrages de F. Ortiz sur la civilisation yoruba, des chants, des proverbes, des prières et des mythes.

J. L. Matory trouve à ce texte une ressemblance surprenante avec les livres et pamphlets publiés au début du siècle au Nigeria, et émet l'hypothèse que N. Angarica aurait eu accès à cette littérature et s'en serait fortement inspiré (2001 : 183-184). Il est certain que les auteurs de manuels lisaient déjà, bien avant les années cinquante, tout ce qui se produisait sur ces sujets : ils font souvent référence au Brésil ou au Nigeria, comparent les termes, les mythes, les façons de faire. Les religieux n'étaient pas uniquement des 
analphabètes, loin s'en faut, et plusieurs d'entre eux étaient membres du Club Atenas ou de loges maçonniques qui mettaient de nombreux livres traduits à leur disposition ${ }^{11}$. De plus, il semble que divers objets et ingrédients liturgiques étaient importés d'Afrique et commercialisés à Cuba par des voyageurs canariens jusqu'à la première guerre mondiale (Cabrera, 1993 : 389-390); il n'est pas impossible que des manuscrits aient circulé par cette voie. En définitive, le mythe de l'hégémonie yoruba, lui-même fortement impulsé par le retour en Afrique d'esclaves libérés brésiliens, entre autres (Peel, 2000), a sans doute nourri à la fois les théories des universitaires et les stratégies locales des pratiquants cubains de la « religion lucumí».

La focalisation des recherches sur les pratiques d'origine yoruba est aussi à mettre en rapport avec l'engouement décrit précédemment pour les polyrythmies « afro-cubaines ». Au-delà de la première étape de découverte que constitue le son, les comparsas $^{12}$ et les tambours utilisés dans certaines cérémonies dédiées aux orichas fascinèrent les intellectuels : " et notez que, quand apparut la batterie du son, le Noir ne nous livrait pas encore les tambours qui constituaient les batteries rituelles », nous dit A. Carpentier (1985 : 218), avant de nous révéler le nom de ces instruments « simplement prodigieux », les batá, et de s'extasier : « ici la batterie est un organisme palpitant, vivant, frémissant. » (idem : 261).

En 1937, F. Ortiz approfondit son engagement et co-fonda la Sociedad de estudios afrocubanos. Il organisa avec des collaborateurs musiciens deux conférencesspectacles pour l'Institution Hispano-américaine de Culture au théâtre Campoamor. Le 14 novembre 1936, «l'orchestre afro-cubain » de Gilberto Valdés présenta pour la première fois dans une salle prestigieuse des batá et de la rumba intégrés à une œuvre artistique originale, précédée d'un exposé musicologique. Les tambours furent fabriqués spécialement pour l'occasion, et la dimension des peaux prévue pour qu'ils puissent s'accorder sur le la et jouer en harmonie avec les autres instruments de l'orchestre. Le 30 mai 1937, ce fut au tour de F. Ortiz de présenter un spectacle à caractère « ethnographique ».

Dans sa conférence introductive, il fustigea ceux qui considéraient encore que la musique des Noirs n'était que bruit et exprima sa honte à l'idée que « ce sont précisément les étrangers qui estiment le plus le trésor esthétique que nous méprisons ici » (1937: 78). Puis il présenta les Yoruba comme le peuple le plus civilisé d'Afrique occidentale, détenant l'exclusivité du trio de tambours batál ${ }^{13}$. Les musiciens, chanteurs et danseurs étaient les informateurs de F. Ortiz, qui les introduisit en tant qu'artistes cubains prodigieux, héritiers d'une prestigieuse tradition familiale, conscients « de leurs devoirs civiques de culture, de tolérance et de cubanité » (idem : 82). Les tambourinaires avaient pour instruction de « retenir leurs rythmes », afin de ne pas provoquer de transes, « sans rapport avec la

11. J. L. Matory remarque l'influence de l'imaginaire maçonnique chez certaines organisations adoratrices d'orisa au Nigeria à la fin du $19^{\mathrm{e}}$ siècle (idem : 178), qui utilisaient les symboles du compas et du tablier.

12. En 1937, ces dernières furent ré-autorisées avec l'appui de F. Ortiz, qui voyait désormais en elles l'expression d'une culture populaire authentiquement cubaine, et pas encore « parasitée » par les américains (1995 : 58 59 , notes d'Isaac Barreal).

13. Tambours bimembranophones et ambipercussifs, à caisson de bois en forme de sablier, fermés, dont la tension permanente est assurée par un cordage de peau. 
leçon présente $\rangle^{14}$. Les costumes et la mise en scène se voulaient sobres pour préserver l'objectivité du propos. Après une seconde partie où les tambours jouèrent seuls, les santeros se relayèrent en bon ordre (oricha par oricha) pour exécuter les chorégraphies et les morceaux « purs et orthodoxes » du rituel.

Le tambourinaire principal (celui qui jouait sur l'iyá - la mère -, l'instrument le plus grand, destiné aux variations les plus complexes), s'appelait Pablo Roche, Okilápkuá, « Bras merveilleux ». Il s'inscrivait dans une lignée de musiciens en rivalité avec d'autres maisons religieuses célèbres, comme le cabildo Changó Teddún, impliqué en 1812 dans une rébellion abolitionniste avortée. Selon Pablo Roche, les batá y auraient résonné pour la première fois à Cuba, mais vers 1830 deux esclaves lucumí tambourinaires initiés appelés respectivement $A \tilde{n} a b{ }^{15}$ et Atandá les entendirent et les déclarèrent «juifs », c'est-à-dire profanes. Ils fabriquèrent alors le premier « vrai » jeu de batá consacrés. Le tambourinaire du Changó Teddún aurait par la suite sombré dans la folie, ensorcelé par ses deux rivaux. Le jeu d'instruments centenaires fut transmis au père de Pablo Roche, « aujourd'hui l'un des plus célèbres olúbatá de Cuba » (Ortiz, 1995 : 74).

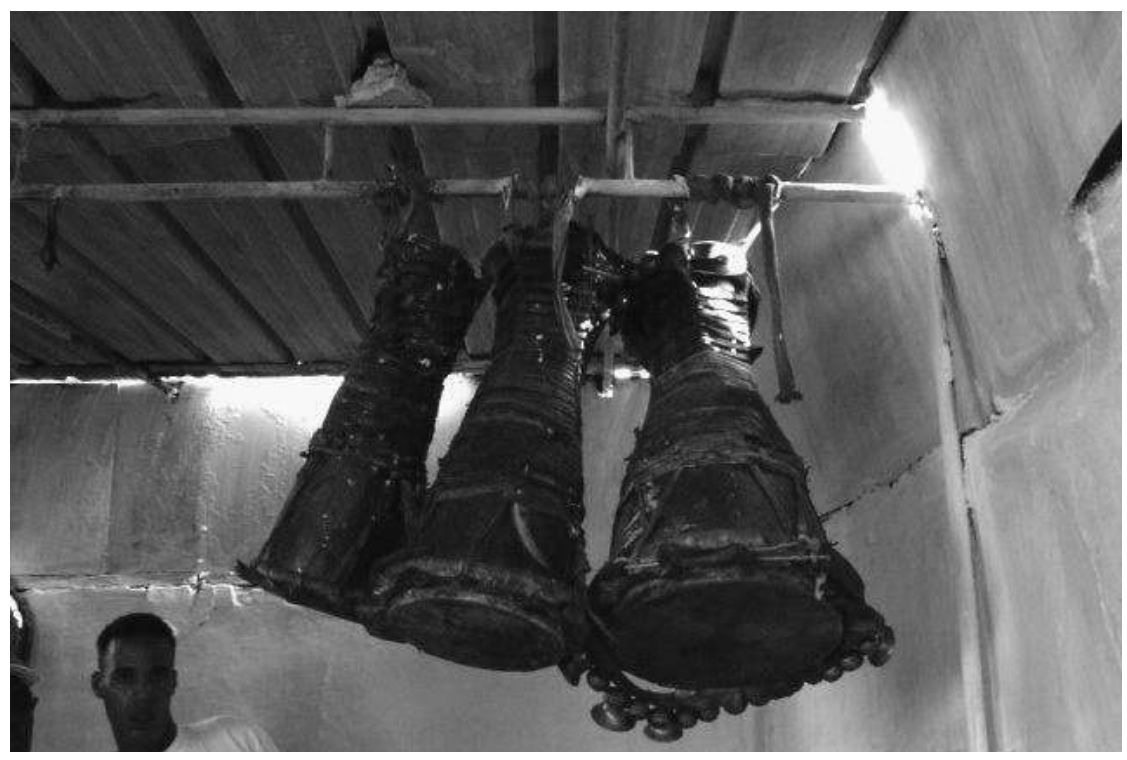

Photo 1 : Tambours batá au repos, chez leur propriétaire (les batá ne peuvent toucher le sol)

(C) Kali Argyriadis).

14. Pourtant, il s'agissait de tambours aberikolá, non consacrés, fabriqués pour l'occasion et donc en théorie non susceptibles de provoquer la venue des orichas. On verra plus loin que ces catégories ne sont pas toujours opératoires.

15. Ce nom signifie précisément «né en Añá». Añá est le nom de la force, du fondement qui vit dans le tambour consacré, qui reçoit régulièrement des offrandes et du sang sacrificiel. Les tambourinaires initiés sont appelés omo añá (fils d'Añá). Le terme olú batá désigne le possesseur d'un jeu de tambours consacrés. F. Ortiz semble confondre les deux termes. 
Dans les années cinquante, F. Ortiz a rapporté ce récit fondateur et légitimant, non sans évoquer quelques autres lignées alliées ou rivales ${ }^{16}$. Ces généalogies, instituées par le texte universitaire, ont acquis depuis valeur de vérité historique. Mais surtout, F. Ortiz a établi une distinction entre vrais et faux batá, en nommant explicitement les détenteurs d'instruments dégénérés, déviants, substitutifs ou irréguliers. Il a dénombré à l'époque seulement onze jeux « orthodoxes » en activité, dont huit à La Havane, et seulement vingt olúbatá dignes de ce nom dans la capitale (idem : 77). Nul doute que cette définition très stricte lui aura été suggérée par ses informateurs, alors confrontés, comme le décrit par ailleurs Ortiz, à la concurrence d'autres musiciens de lignées rivales, ou non initiés mais présentant l'avantage pour les religieux de se faire rémunérer à meilleur marché.

En effet, si la production de rythmes spécifiques est indispensable pour faire venir les orichas, il est aussi nécessaire de présenter en public chaque nouveau santero (iyawó) aux tambours pour ratifier l'initiation. Pour ce faire, divers types de membranophones sont utilisés, présentés ou non comme traditionnels, appelés ou non batá. Dans les années quarante, les tambourinaires ne vivaient pas encore de leur art, et étaient obligés d'exercer par ailleurs d'autres métiers. Leur besoin de reconnaissance n'en était pas moins fort: une vingtaine d'entre eux, partageant la même vision de la tradition en matière de batá, alarmèrent l'universitaire en attirant son attention sur l'inéluctabilité de leur disparition, et donc de "l'affaiblissement de l'orthodoxie yoruba». Les musiciens qui jouèrent au Campoamor (Pablo Roche, Trinidad Torregrosa) et deux de leurs associés (Miguel Somodeville, José Valdés Frías) rédigèrent et distribuèrent en 1950 une Circulaire aux santeros (reproduite dans Ortiz, 1995 : 72). Le tract rappelait aux religieux leurs devoirs envers les tambourinaires recrutés : les nourrir copieusement et avec des marques de respect, leur verser $50 \%$ des offrandes monétaires faites aux possédés, assurer leur transport et leur logement le cas échéant. Mais surtout, il revendiquait explicitement l'hégémonie rituelle des auteurs : «La présentation de n'importe quel yaguó [iyawó] n'aura aucune valeur si elle n'est pas effectuée devant un Aña fondamental, parmi ceux qui sont connus à ce jour à la Havane et à Matanzas. ». Ainsi, la validité de l'initiation d'un rival pouvait être remise en cause : le monde religieux n'a sans doute pas manqué d'être ébranlé par cette affirmation de pouvoir venue de personnes désormais auréolées du prestige de la reconnaissance universitaire. «Ceux qui sont connus » avaient leurs noms complets écrits dans des livres scientifiques, ils se produisaient sur des scènes nationales et ils étaient les informateurs des chercheurs et des artistes.

La méfiance envers ces derniers et la loi du silence laissèrent de fait progressivement la place à d'autres stratégies. En 1942, F. Ortiz ouvrit un séminaire d'ethnographie cubaine à l'université de La Havane, et devint membre d'honneur du Club Atenas. Il prononça à cette occasion une conférence intitulée Pour l'intégration cubaine des blancs et des noirs, où il évoquait la réaction de ses informateurs face à ses recherches : «A l'hostilité pleine de préjugés qu'avaient pour moi les gens de couleur ont succédé ensuite le silence prudent, l'attitude indécise et une respectueuse courtoisie, mélange de timidité, d'excuse et de demande de faveur. » (cité par García Carranza et al., 1996 : 11).

16. Celle par exemple de Martín Oyádiná, dont les fameux tambours, confisqués par la police, étaient exposés au Musée national de La Havane, et celle d'un sculpteur appelé Adofó, dont est issu un autre informateur de F. Ortiz et allié de Pablo Roche, Miguel Somodeville. 
Ces requêtes concernaient sans doute l'arrêt des persécutions policières et l'acquisition d'une légitimité religieuse via la validation des réputations et des filiations rituelles par le texte universitaire.

Les manuels rédigés dans les années cinquante par les santeros témoignent également de ces objectifs ainsi que des rivalités et alliances nouées par les religieux, qui se définissent toujours par rapport au modèle canonique du batá, seul instrument rituellement adéquat. La santera María Antoñica Finés n'hésite pas, par exemple, à évoquer la possibilité d'utilisation d'autres instrument antérieurs, comme les gros hochets-sonnailles agües (1990 : 43). Elle cite tout de même F. Ortiz et la filiation qu'il a décrite mais récidive en rappelant que les tambours bembé « furent les premiers à être joués ici à Cuba. » (idem : 75). Or Ortiz insiste pour les cataloguer comme profanes, bien qu'à l'évidence les orichas se manifestent à leur appel. Il se rabattra plus tard sur l'expression «semi-profanes » (1981: 380). Un autre auteur religieux, Jesús Torregosa (1990 : 256) tient à afficher son appartenance à la lignée fameuse dans sa transcription de la prière liminaire destinée à rendre hommage aux tambourinaires défunts et à leur demander la permission de commencer le rituel; on reconnaît sans peine les noms moult fois cités : « (...) ño Juan Andrés, Iba Añabi agia bata, Tanda adechina, Iba agüegüi Iche Ilú - Le premier charpentier qui fit des Batá, Iba Atanda, Iba Ifatola ${ }^{17}$, Iba Ado Foó ». Il a également le souci de comparer différentes tailles de batá : celles de ses instruments personnels, celles issues des « mesures officielles des tambours de fondement », celles de F. Ortiz et celles des tambours de Oya Dina Trunda, observé dans les vitrines du Musée National en 1936 (idem : 268).

De fait, la multiplicité des références et la plasticité sont de règle en matière d'instruments rituels, et le discours de l'orthodoxie sous-tend bien plus les luttes de pouvoir que la pratique elle-même. F. Ortiz a établi des hiérarchies sans pour autant négliger une description exhaustive de toutes les variantes. Son texte regorge de descriptions et d'illustrations d'instruments de toutes sortes, utilisés selon lui par méprise mais avec foi et sincérité dans les cérémonies dédiées aux orichas. On découvre ainsi profusion de tambours appelés batá mais de forme et de montage différents (" dégénérés », 1995 : 119), dont certains rappellent les grosses caisses de fanfare, ou résultent de l'ingéniosité de leur concepteur désireux d'échapper aux confiscations, comme celui de Papá Silvestre, monté sur une caisse de bois qui sert aussi de siège (idem : 130).

La frontière entre sacré et profane est bien souvent ténue en santería, et reste plus une question d'intention que de répertoire musical. A l'instar de F. Ortiz, plusieurs auteurs font état de la possibilité de se contenter de simples frappements de mains, voire même de musique enregistrée ou diffusée à la radio. Certes, les rythmes spécifiques aux orichas sont préférés, mais à défaut de musiciens capables de les exécuter correctement on n'hésite pas à utiliser le danzón ou le cha-cha-chá (Cuellar Vizcaíno, 1950). Dans d'autres cas toujours d'actualité, en l'honneur des orichas féminins Yemayá et Ochún on fait appel à un orchestre de violon, guitare, claves, maracas, chekeré, bongó et tumbadora. On y chante et danse aussi bien les airs à la mode, à thème religieux, que les chants d'orichas les plus connus ou des morceaux d'inspiration spirite composés en espagnol. Enfin la rumba, qu'elle soit jouée sur des caisses préparées à cet effet (cajones) ou des

17. Ifábola est le fils d'Atandá selon Ortiz, 1995 : 70. 
tumbadoras est abondamment utilisée dans les cérémonies spirites et paleras, et il arrive que les orichas se manifestent sur son rythme ${ }^{18}$.

Le répertoire des batá est lui aussi soumis à des variations et à des innovations. Miguel Somodeville se plaignait à F. Ortiz dans les années cinquante de la tendance qui poussait les musiciens à privilégier le plaisir des danseurs au détriment du strict respect de la tradition, selon laquelle la polyrythmie devait reproduir le langage tonal des Yoruba. A défaut d'auditeurs capables d'apprécier une telle performance, les olubatá se virent obligés de jouer en intégrant le rythme de la rumba, car «les gens payent mieux les tambourinaires qui battent leur instrument avec profanation » (Ortiz, 1995 : 51). L'idée d'un corpus de morceaux fermé et normé est infirmée par les descriptions musicologiques très fines de F. Ortiz, qui fait état de possibilités d'improvisations variées (cassures, combinaisons de rythmes d'appels, liaisons, «conversation » entre le tambourinaire et le danseur ou le chanteur...). Des compositions nouvelles ont été créées par Trinidad Torregrosa vers 1940 (Ortiz, 1981 : 93). Pablo Roche, quant à lui, a restauré un vieux morceau tombé en désuétude (idem : 356). La valeur de traditionalité des morceaux de batá s'acquiert donc dans un cadre politique et historique précis et constitue plus une innovation qu'une reproduction fidèle de modèles anciens.

La variété des formations instrumentales présentes dans les cérémonies santeras est-elle le reflet, comme l'affirme F. Ortiz, d'un appauvrissement culturel ${ }^{19}$ ? Plusieurs sources africanistes incitent au contraire à y voir une continuité logique et un processus contemporain parallèle. P. Verger et G. Rouget ont par exemple observé au Dahomey en 1952 un orchestre de bàtá qui s'écartait manifestement de la forme canonique décrite par W. Bascom ou F. Ortiz : leur forme était bombée et non clepsydrique, un quatrième tambour avait été ajouté, ainsi qu'une trompe traversière décrite comme essentielle au déclenchement des transes. G. Rouget en conclut qu'il s'agissait d'une «forme fruste, paysanne de l'instrument » (1965: 78). Il s'en remettait pour cela à un informateur local très engagé, chrétien et militant actif de l'affirmation de la traditionalité de la musique yoruba, Laoye I, le Timi (chef) d'Ede en pays Oyo. Les ambitions de ce dernier semblent claires quand il affirme que «l'art de jouer des bàtá est en disparition au Nigeria, sauf à Ede » (cité par Leymarie, 1996 : 12). Laoye I prit en charge les ethnologues comme il le faisait pour les étrangers de passage (Rouget, 1965 : 76). Toutefois, il ne s'en tint pas uniquement aux bàtá (plutôt spécifiques aux cultes à Changó et aux ancêtres dans la région d'Oyo) et fit état d'une immense variété de jeux de tambours et d'idiophones selon les régions et les usages, qui pouvaient se combiner, se mélanger, ou s'ajouter pour former des orchestres hétérogènes (Laoye, 1961 : 23). G. Rouget compara toutes les données qui étaient à sa disposition, et conclut fort judicieusement : «A Cuba comme en Afrique les faits sont extrêmement contradictoires » (1965: 84). Cela ne l'empêcha pas de contribuer explicitement à la fixation et à l'esthétisation du répertoire des bàtá, dans un souci typologique d'élaboration d'une « esthétique scientifique de la musique ». Comme F. Ortiz, il œuvra pour la valorisation auprès de ses pairs d'une pratique artistique qu'il

18. Les observations de terrain actuelles corroborent les anecdotes racontées à ce sujet par les santeros âgés; voir par exemple l'interview de Mario Dreke « Chavalonga », célèbre rumbero octogénaire (Mestas, 2000 : 40).

19. Dans son essai écrit en 1946, El engaño de las razas, ce dernier proposait de supprimer le terme race et de le remplacer par le mot culture. Cette notion, chez F. Ortiz, est profondément essentialiste. 
appréciait : « On sait à quels sommets de l'art ont atteint les bronzes, les ivoires, les bois sculptés des Yoruba. Dans le domaine de la musique, leur chant sacré atteint souvent à des réussites comparables. \ (idem : 68). Comme son collègue cubain, il opéra explicitement des tris : il choisit les deux pièces principales du disque qui accompagnait son article pour leur « grande beauté musicale » et précisa qu'il avait tronqué une autre partie car elle était « mal chantée » (idem : 63).

S'il y a bien un point commun entre l'Afrique de l'Ouest, le Brésil et Cuba dans les années cinquante, c'est qu'on y retrouve les mêmes anthropologues, alors très engagés (y compris parfois spirituellement) pour la reconnaissance de l'existence de survivances africaines prestigieuses en Amérique. Outre W. Bascom, qui affirma avoir rencontré à Cuba en 1948 des personnes maîtrisant de façon évidente le yoruba, le fon ou le kikongo (Bascom, 1952) et s'intéressa par la suite au système divinatoire ifá, A. Métraux et P. Verger accompagnèrent L. Cabrera en 1956 chez «ses Noirs », pour reprendre l'expression ambiguë de la chercheuse ${ }^{20}$. Ils enregistrèrent ${ }^{21}$ et photographièrent ensemble une cérémonie en l'honneur de Yemayá à la lagune San Joaquín. L'échelle de valeur des modestes informateurs de L. Cabrera se trouva complètement bouleversée lorsqu'elle leur expliqua que les respectables visiteurs étrangers étaient adeptes de Yemayá Afreketé et les accompagnaient pour lui rendre tribut : «Quand nous avons expliqué à Ma Francisquilla que Verger était français... un Français babalawo ! qui allait à Lagos, au Dahomey, en Sierra Leone, qui connaissait Abeokuta, Ibadan, Oyo « la terre d'Olokun », tous noms familiers pour elle, nous nous souvenons de ses cris d'étonnement, de ses sursauts, du sourire de satisfaction que découvrirent ses fortes gencives édentées et des coups qu'elle se donna sur le front: Modu Modu! Musiú francés babalawo! Eh! Franchute awó, mía qué cosa! » (cité par Bolivar, del Río, 2000 : 81-82).

La fin des années quarante marqua aussi le moment où les cultes dits afro-cubains, ayant acquis une certaine visibilité (à défaut d'une reconnaissance pleine et entière), devinrent à la mode : publications, disques et spectacles autour de ce thème abondaient à cette époque ${ }^{22}$. A partir de 1948, F. Ortiz et le musicologue Gaspar Agüero commencèrent

20. Il s'agissait des ouvriers agricoles qui travaillaient et vivaient sur la propriété de son amie Josefina Tarafa (village Pedro Betancourt, région de Matanzas). Malgré l'abolition de l'esclavage, le paternalisme était encore de mise dans l'île à cette époque. J. Tarafa finança les enquêtes de L. Cabrera sur ses terres, elle lui procura du matériel photo et audio de pointe, ainsi qu'un mini-van climatisé (Bolívar, del Río, $2000: 33$ ).

21. Les disques font partie de la collection Música de los cultos afrocubanos en Cuba, qui s'arrache aujourd'hui à Miami, en tant que référence traditionnelle incontestable.

22. En 1948, la chanson de Celina González, Que viva Changó est un énorme succès. D'autres enregistrement dits « afro » lui succèdent, portés par les artistes en vogue de l'époque : Bola de Nieve, Mercedita Valdés et Celia Cruz, accompagnées par les « tambours rituels » de Jesús Pérez . Benny Moré, Miguelito Cuní, Arsenio Rodríguez (qui vit à New York) et d'autres encore ne manquent pas de faire allusion à la santería ou au palo dans les chansons qu'ils interprètent. Dans le cadre de la naissance du latin jazz évoquée plus haut, l'inspiration lucumí ou congo est constamment mise en avant. En 1955, le groupe de rumba Los muñequitos de Matanzas sort son premier disque : Guaguancó matancero. D'autres suivent sous divers labels cubains ou américains, comme ceux de Celeste Mendoza, ou le disque de « chants rituels yoruba », produit par la compagnie de disques cubaine Panart, intitulé Santero. Odilio Urfé, le directeur de l'Institut musical de recherches folkloriques, présente en 1956 une anthologie de rumbas célèbres, qui sont aujourd'hui des classiques incontournables, comme Consuélate como yo, Malanga, ou Ave María morena. 
à transcrire, à l'instar du jeune Argeliers León, les rythmes et les chants du répertoire des batá, suivis bientôt d'autres instruments de percussion. Ils furent aidés par un nouvel omo añá, Raúl Díaz, " virtuose érudit et compétent » qui avait de plus le mérite de connaître le solfège (Ortiz, 1993 : 269). Parallèlement, et malgré l'indignation des chercheurs, les cabarets proposaient des danses lucumí à connotation sexuelle très renforcée et des « rumbas à quatre pattes », tandis que des cérémonies-spectacles étaient organisées à grand renfort de journalistes invités. S'y distinguèrent tout particulièrement l'étoile montante Mercedita Valdés, accompagnée par les batá de Jesús Pérez, qui n'était autre que le jeune joueur d'okónkolo (le plus petit batá) qui avait accompagné Pablo Roche au théâtre Campoamor en 1937. Des personnages politiques importants, comme le maire de La Havane, le ministre de la défense, et même le président de la république, se montrèrent dans les cérémonies. Enfin dans le même temps les santeros et spirites demandaient de plus en plus de permis à la police pour organiser leurs fêtes, et certains journalistes les soutinrent dans leurs revendications : acquérir un statut légal, en tous points similaire à celui des catholiques (Cuellar Vizcaíno, 1948).

L'exploitation marchande du répertoire liturgique afro-cubain, « qui cherche à profiter de la valeur mercantile de l'exotisme des cultes africains », a été fortement décriée par F. Ortiz (1981 : 149). Pourtant, c'est aussi sur la scène de l'industrie du disque et du spectacle que se sont jouées les luttes hégémoniques. Comme l'accusation de sorcellerie, l'accusation d'appât du gain a sous-tendu les stratégies des musiciens rituels rivaux. Ces derniers ont d'une certaine manière échappé en cela au contrôle des intellectuels, qui ne se tarissaient pas d'éloge sur l'œuvre d'un Amadeo Roldán mais rejettaient avec dégoût les innovations artistiques d'une Celina González. Or les omo añá avaient à chœur de dépasser le cadre restreint de leur réseau de relations local et de s'affirmer sur les deux plans : reconnus comme artistes par les universitaires, le public religieux dont ils voulaient gagner le respect n'en était pas moins un public populaire. Les mérites de Jesús Pérez sont soulignés lorsqu'il joue aux côtés du "maître » Pablo Roche; ils sont vus d'un tout autre œil quand il se produit aux côtés de Celia Cruz : pour l'intéressé, il n'y a pourtant ni contradiction ni profanation, puisque dans un cas comme dans l'autre il n'a pas utilisé d'instruments consacrés. Cette volonté de passage d'un registre à un autre (religieux, scientifique, artistique, commercial), menée jusqu'au bout dans le contexte révolutionnaire, lui a d'ailleurs apporté, à lui comme à d'autres, une reconnaissance nationale et internationale indiscutable.

\section{Théâtralisation du folklore}

Malgré le succès commercial de la musique afro-cubaine, malgré l'intérêt qu'elle suscitait auprès des intellectuels, la pratique religieuse dont elle était issue restait largement stigmatisée. Un babalao de 60 ans tient à le rappeler : «Avant la révolution, être pratiquant de ces religions cela signifiait que l'on pratiquait une religion de caractère africain, c'était s'humilier sur le plan du statut social. On était mal vu non par l'élite mais par les couches moyennes de la société » (Mercier-Baláz, Pinos, 1994). Lorsque les troupes de Fidel Castro prirent le pouvoir en 1959, elles affichèrent très vite une volonté de redonner une dignité à ceux qui étaient marginalisés : gens « de couleur », prostituées, petits paysans, ouvriers... Les religieux furent considérés comme faisant partie des couches de population les plus pauvres, ce qui leur confèra le droit à l'élaboration de 
toutes sortes de projets associatifs ${ }^{23}$. Des chercheurs et artistes éminents comme Odilio Urfé, Argeliers León, ou encore René Depestre se firent l'écho de leurs revendications et érigèrent ces pratiques en tant que symbole de la résistance culturelle du peuple opprimé (Argyriadis, 1999 : 263-266). La volonté politique du gouvernement envers la religión était ambiguë : glorifiée dans ses qualités esthétiques, contre-culturelles et identitaires, elle restait violemment condamnée pour son mysticisme, qui faisait insulte aux acquis sociaux de la révolution.

Libérée du «joug impérialiste», la jeune nation voulait se donner les moyens d'affirmer une identité brimée malgré l'acquisition de l'indépendance en 1898. Cet objectif rejoignait les idées de F. Ortiz ou des afrocubanistes (A. Carpentier et N. Guillén s'engagèrent d'ailleurs pleinement dans la cause révolutionnaire). Dès 1950, le « troisième découvreur de Cuba $»^{24}$ trouvait lamentable que les richesses populaires et folkloriques de l'art musical et littéraire cubain ne soient pas enseignées dans les conservatoires et les académies (Ortiz, 1993 : 98), et prônait la création d'une Société de musique afro-cubaine ou de Folklore musical de Cuba, ayant un double objectif, scientifique et esthétique (Ortiz, 1981 : 587). Son souhait fut exaucé en 1960 avec la création du Centre d'études du folklore du Théâtre national de Cuba.

Son assesseur, Argeliers León, précisait d'emblée : «Il s'agit maintenant de prendre les expressions de notre peuple, et de les offrir à nouveau sans les dévoyer. Il est certain qu'une grande partie du folklore cubain provient de lointains apports africains qui se trouvent encore aujourd'hui intimement connectés à un lacis emmêlé de croyances. Ici nous nous éloignons de l'aspect intime et particulier du religieux et nous essayons de présenter les valeurs pures de chant, de danse et de poésie » (León, 1961a : 5). La création de spectacle théâtraux fut conçue en osmose avec la recherche et la publication. Avec l'appui financier de l'UNESCO, 35 élèves, parmi lesquels de futures célébrités comme Miguel Barnet, Rogelio Martínez Furé ou Rafael López Valdés travaillèrent en étroite relation avec de vieux informateurs à vérifier l'authenticité des matériaux et à en classer les variantes. A. León insistait néanmoins pour que le terme folklore soit entendu dans le sens d'une pratique vivante et authentique, son objectif et celui des ses collaborateurs étant de « s'approprier notre propre culture » (idem : 6). Le sens qu'il donnait à cette dernière phrase ne faisait pas l'unanimité, puisque le Centre d'études du folklore du TNC fut rapidement accusé de privilégier l'inspiration afro-cubaine au détriment de l'inspiration guajira, étudiée par la femme d'A. León, María Teresa Linares.

En décembre 1961, le Centre fut dissous et remplacé par l'Institut d'ethnologie et de folklore. Sous la direction d'A. León, l'IEF se consacra désormais exclusivement à la recherche scientifique ${ }^{25}$, tandis que le développement artistique était confié en 1962

23. A condition qu'ils soient pro-révolutionnaires : la plupart de ces initiatives n'ont pas résisté au durcissement idéologique de la fin des années soixante.

24. C'est ainsi que F. Ortiz est désigné dans les incessants hommages qui lui sont rendus depuis sa mort en 1969. Le premier découvreur est Christophe Colomb, et le deuxième Alexander von Humboldt.

25. L'IEF fut aussi chargé d'assurer la conservation du patrimoine, en créant un musée d'ethnologie. Les méthodes employées pour récupérer certains objets religieux n'ont parfois rien eu à envier aux confiscations policières d'antan, aux dires de nombreux religieux qui se sentent aujourd'hui spoliés : prêts non rendus, autels saisis arbitrairement suite au décès de leurs propriétaires pourtant dévoués à la cause révolutionnaire (Fernández Robaina, 1994 : 64), maisons des exilés pillées. L'ensemble se trouve aujourd'hui pour une grande part au Musée historique de Guanabacoa, aux côtés de la collection personnelle de F. Ortiz. D'autres musées municipaux ou régionaux ont vu le jour depuis. 
au tout nouvel Ensemble folklorique national. Le gouvernement souhaitait unifier enfin la nation sur la base de valeurs propres et former une conscience nationale « de premier ordre » : l'Institut se donna donc pour but de « déterminer la présence populaire dans l'intégration culturelle de Cuba » (León, 1961b : 34). Derrière cette expression un peu floue, il faut comprendre le rôle attribué de façon explicite aux sciences humaines dans la Cuba socialiste : "connaître et améliorer la société »" ${ }^{26}$ le Parti se chargeant de l'analyse des résultats, de l'opportunité de leur publication et de leur application possible.

Pendant quelques années encore, les études afro-américaines mobilisèrent l'attention des ethnologues, qui publièrent de nombreux articles dans la revue Etnologia y Folklore. Elles laissèrent ensuite progressivement la place aux travaux sur le folklore rural. La fin des années soixante était au matérialisme sans concessions, et il n'était pas bon d'afficher ses croyances religieuses (Argyriadis, 1999 : 267). Le Centre d'anthropologie recentra ses efforts sur les provinces, et opta pour de grandes études à échelle nationale comme le Calendrier de fêtes traditionnelles, suivi en 1976 du projet de création d'un Atlas ethnographique qui tint les chercheurs cubains en haleine pendant plus de vingt ans ${ }^{27}$.

Les religieux disposaient cependant d'un autre espace d'expression pour donner une image valorisante de leurs pratiques. Tandis que le gouvernement interdisait les cérémonies religieuses publiques comme la procession de la Vierge de Regla, avatar catholique de l'oricha Yemayá, où se produisaient des batá, l'Ensemble folklorique national se mettait en place. En 1962 les informateurs des ethnologues furent chargés de sélectionner une soixantaine de musiciens, chanteurs et danseurs parmi les 150 volontaires présents. Jesús Pérez Fuentes, l'okónkolo de Pablo Roche en 1937, fut par exemple chargé de la sélection des percussionistes, aux côtés de Trinidad Torregrosa et d'autres encore. Il n'est pas aujourd'hui un seul artiste du répertoire afro-cubain qui ne fasse systématiquement référence à ces « grands ancêtres » que sont les religieux qui ont créé les comparsas et collaboré avec F. Ortiz dès 1937 ou avec L. Cabrera en 1956, s'enorgueillissant d'avoir appris avec eux (pour les plus âgés) ou avec leurs élèves, membres éminents de l'Ensemble folklorique national ${ }^{28}$.

Les débuts de la troupe furent marqués par des confrontations parfois tragiques qui témoignent de tensions et de préjugés de race et de classe persistants ${ }^{29}$. Dans un entretien accordé à K. Hagedorn (2001 : 154), M. T. Linares accuse les artistes en tournée de crimes

26. Entretien officiel avec un fonctionnaire du Bureau d'attention aux affaires religieuses du Comité Central du Parti Communiste Cubain, avril 1993.

27. Y sont répertoriés essentiellement les manifestations matérielles de la culture rurale comme l'artisanat, l'habitat, l'outillage, le mobilier, ainsi que les contes, chants, danses et « fêtes populaires traditionnelles », parmi lesquelles les rituels occupent une place minoritaire, notamment du fait que ne sont pas pris en compte ceux qui concernent des groupes non institutionnalisés, c'est-à-dire l'immense majorité des réseaux de parenté rituelle à cette époque (voir Fiestas populares..., 1998).

28. Sauf bien entendu leurs rivaux de même génération exilés aux Etats-Unis, qui les accusent d'avoir « trahi la religión » pour échapper aux persécutions du régime et au soupçon de délinquance (Hagedorn, 2001 : 152).

29. Présents à la même époque dans la plupart des nouvelles institutions révolutionnaires, comme l'IEF qui promut au poste de chercheur ou d'auxiliaire de recherche des personnes considérées par leur rivaux comme « des paysans ou des ouvriers incultes, grossiers, bons à rien, tout juste capables de dénoncer leurs collègues ou leurs informateurs à la Sécurité de l'Etat ou de régler leurs comptes au couteau ou au pistolet ». 
qu'elle qualifie de vulgaires et associe à leur couleur de peau, à leur milieu d'origine et à leur manque d'éducation : ivrognerie (et donc absence de discipline professionnelle), menus larcins, escroqueries, proxénétisme, rejet de la nourriture étrangère ... En 1965, elle assumait la direction de la troupe et entra en conflit avec la danseuse Nieves Fresneda, qui refusait de créer une chorégraphie basée sur une cérémonie d'initiation, jugée trop « sacrée », et lui insinua de façon méprisante que beaucoup d'autres santeras pourraient prendre sa place. M. T. Linares déplore en outre l'existence, à cette époque, de « deux structures sociales distinctes » au sein de la troupe, la plupart des membres étant en réalité choisis par les artistes-informateurs fondateurs sur des critères de filiation rituelle. Elle résume son point de vue en affirmant : « ce n'était pas une institution culturelle, c'était un groupe de santeros » (idem : 155).

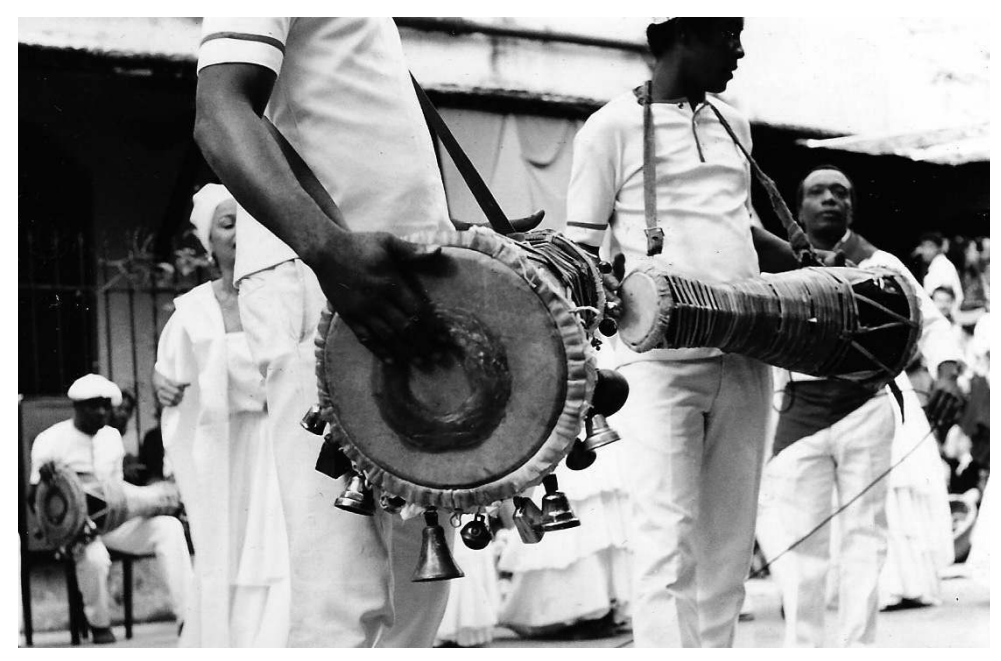

Photo 2 : Tambours batá joués pendant un Sábado de la rumba, Conjunto Folklórico nacional, 1992.

L'Ensemble folklorique national s'est néanmoins produit dans toute l'Europe, toute l'Amérique, et plusieurs pays d'Asie et d'Afrique, où il a remporté un grand succès. Ses membres reçoivent désormais des salaires équivalents à ceux de la troupe du Ballet national (ce qui n'était pas le cas au début). Il a également assis les effets de son impact local en produisant des disques et des documentaires qui passent à la télévision nationale. Ses artistes ont participé à des pièces de théâtre engagées dans la cause anti-raciste, ainsi qu'à plusieurs films où les personnages d'Africains, de descendants d'esclaves ou de religieux jouent des rôles de premier plan, comme La última cena de T. Gutiérrez Alea. Le système d'enseignement artistique cubain (Ecoles d'art et Institut supérieur d'art) a peu à peu inclut dans ses programmes de formation les répertoires « populaires » et «folkloriques », qui ont pour professeurs les musiciens, chanteurs et danseurs de l'Ensemble.

Depuis 1982, la troupe présente ses œuvres les samedis après-midi dans le patio de son siège, en plein quartier résidentiel (Vedado) et ce malgré les réactions hostiles du voisinage. Les spectacles du Sábado de la rumba sont présentés de façon didactique et 
participative : le public, debout et en cercle, est invité à s'investir oralement (identification des pièces et des répertoires, apprentissage des paroles des chants et de leur signification) et physiquement (apprentissage des rythmes par frappement de mains, improvisations dansées), à rivaliser de virtuosité, sous le regard jubilatoire des « anciens » qui cautionnent le tout par leur présence, leur participation et leur approbation. Entre spectacle, cours, fête et cérémonie religieuse, le Sábado de la rumba a été et reste encore ${ }^{30}$ un grand moment d'affirmation d'une identité africaine valorisante, partagé avec enthousiasme par des individus aux couleurs de peau et aux origines sociales variées. De façon générale, les productions très vivantes de l'Ensemble folklorique national ont contribué à attiser la curiosité des personnes ne connaissant pas la religión et suscité de nombreuses vocations à partir du milieu des années 80 .

Ces effets ont largement dépassé le projet théorique général de «théâtralisation du folklore » (Guerra, 1982 : 5-21). Avec les travaux pionniers de M. T. Linares, les efforts se sont essentiellement portés sur le répertoire paysan guajiro, qu'on supposait apte à renforcer le sentiment communautaire, ou sur les groupes urbains à caractère profane ou associatif, riche d'un passé résistant historiquement attesté (comparsas, vieux cabildos, tumba francesa...). La révolution a stimulé en ce sens la production artistique, organisé des festivals, proposé des Ecoles nocturnes de dépassement artistique, ou soutenu la création de groupes folkloriques, mais elle n'a pas encouragé pas l'adhésion religieuse. Pourtant cette dernière a presque invariablement accompagné l'appréciation esthétique des amateurs de musiques et de danses dites afro-cubaines, car les acteurs principaux de la valorisation de ce répertoire sont devenus les religieux eux-mêmes, formés par des techniciens de la mise en scène du folklore. Transportés par l'euphorie de danses qui ont été créées précisément afin de produire cet effet, ils ne résistent jamais au désir d'entraîner le public sur la scène, et se surpassent lorsqu'ils trouvent du répondant. En cela réside sans doute toute l'ingéniosité et l'efficacité de leur contribution à un dispositif au départ normatif et peu spontané.

La théâtralité folklorique populaire est en effet théorisée et enseignée de façon très méthodique à Cuba. Elle comprend quatre niveaux (idem) : le premier, appelé « rituel », où forme et contenu sont indissociables, le deuxième, nommé « projection folklorique », qui consiste en une simple répétition de la forme, le troisième, dit de «théâtralisation folklorique », producteur « d'effets esthétiques capables de sensibiliser émotivement ou intellectuellement le public », et enfin le dernier, la «création artistique », qui constitue le niveau supérieur à atteindre. L'artiste en effet, en recréant une œuvre, lui donne un nouveau contenu et agit sur la société, il « réinvente la tradition ». Pour aboutir à cet objectif ultime, il est essentiel néanmoins de porter une attention particulière au troisième niveau. La technique est ensuite détaillée : il faut styliser et soigner les effets, pour éviter la monotonie concomitante à la vision passive d'une cérémonie ou d'une fête. On peut par exemple utiliser la symétrie dans les chorégraphies, présenter plusieurs danseurs au lieu d'un, raccourcir les morceaux, intégrer les musiciens sur la scène de façon plastiquement fonctionnelle, soigner les décors et les costumes ou encore ménager des points culminants. Il importe également de rester au plus près de la « vérité folklorique » : les représentations sont toujours assorties de commentaires didactiques identifiant les formes syncrétiques,

30. Pour une analyse ethnographique actuelle de ce spectacle (rebaptisé Gran Palenque) en temps que lieu-clé du tourisme culturel, voir Argyriadis 2005. 
anciennes ou contemporaines et écartant avec dégoût les avatars populistes et mercantiles pré-révolutionnaires.

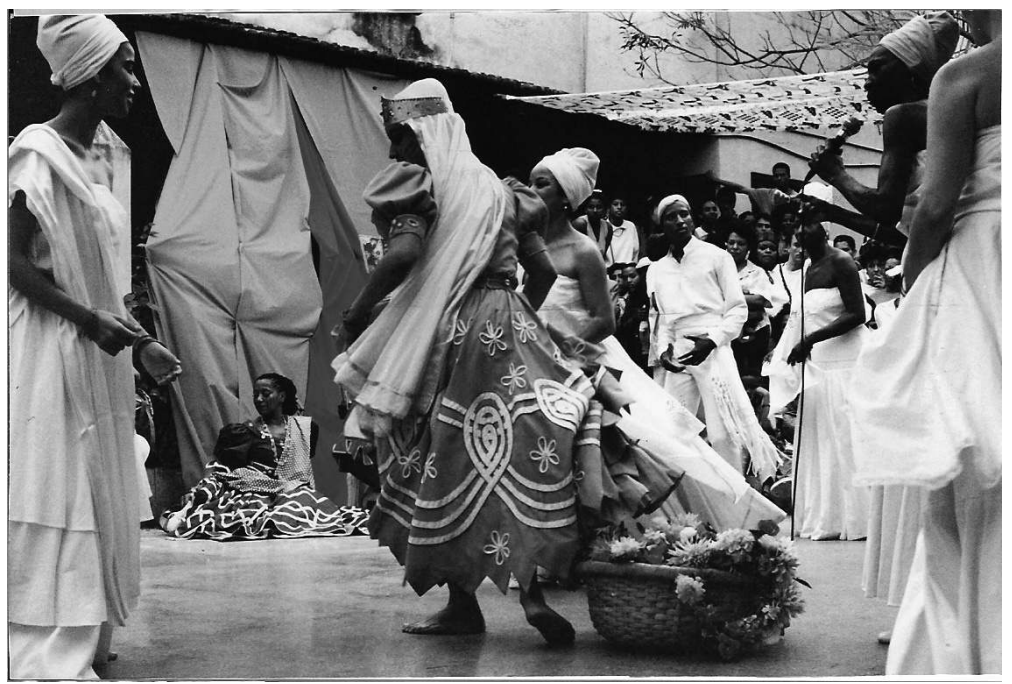

Photo 3 : Danse d'Ochún, Sábado de la rumba. Conjunto Folklórico nacional, 1992.

Les mises en scènes de l'Ensemble folklorique national s'inspirent de ce modèle. Une cérémonie santera est par exemple exécutée par des danseurs vêtus de blanc, évoluant en cercle face aux batá. A l'appel de leurs chants spécifiques, les solistes, déjà costumés et assis devant les offrandes dans un espace représentant l'autel, se succèdent magistralement en jouant le rôle d'un oricha se manifestant à travers le corps d'un adepte et investissent tout l'espace. Chaque morceau dure quelques minutes tout au plus, et fait suite à un autre dans un ordre précis qui correspond au moment où les divinités sont saluées et appelées à tour de rôle (décrit par F. Ortiz, 1981 : 294-352). Or dans une cérémonie les possessions n'ont jamais lieu à cet instant-là, hormis celles, très furtives, des iyawó venus se présenter aux tambours dans leur costume d'apparat. Elles interviennent plus tard, parfois seulement au bout de plusieurs heures, alors que chants et rythmes se succèdent de façon contingente à la situation rituelle (motivations du commanditaire, appréciations in vivo des musiciens, quantité et nature des transes...).

Tout comme F. Ortiz profite, au cours de sa rédaction, de cette partie introductive de la cérémonie où tous les orichas sont cités pour décrire leurs danses, leurs rythmes et leur gestuelle particulières, la théâtralité folklorique condense ici les moments forts et présente l'essentiel des performances possibles au sein d'un répertoire. Ce faisant, elle participe à la fixation de celui-ci, et influe sur la pratique rituelle ${ }^{31}$. La gestuelle

31. Lors d'un Sábado de la rumba en 1992, une vieille santera demandait au présentateur pourquoi Obá avait les mains sur les oreilles. La réponse (évidente pour ceux qui connaissaient l'article de W. Bascom se rapportant à ce mythe) était qu'Obá, trompée par sa co-épouse Ochún, s'était coupé les oreilles pour les intégrer à la soupe de son mari Changó, provoquant la répulsion de celui-ci. Des religieux qui ne la connaissaient pas découvraient la danse d'Ogué, maître des troupeaux, avec un doigt sur chaque tempe figurant les cornes. D'autres s'étonnaient de voir danser Orula, puis observaient attentivement. 
des seize orichas présents dans la suite décrite ci-dessus est précisée. Comme elle est censée traduire les paroles du chant correspondant, les chercheurs attachés à l'Ensemble folklorique compilent les informations et utilisent les sources bibliographiques africanistes en cas de lacune. Les paroles des chants sont notées à partir des exécutions originales des informateurs-professeurs, correctement orthographiées et corrigées si besoin est.

Lors des répétitions, les danseurs s'entendent sans cesse répéter que chaque oricha est en quelque sorte un archétype, et que chacune de ses danses consiste en une pantomime. Ils doivent donc peaufiner leur jeu d'acteur, ne pas se contenter de gestes corrects, mais vivre leur rôle dans toute leur manière de se mouvoir. Les chorégraphies deviennent ainsi accessibles aux profanes. Ochún se passant les mains sur tout le corps en roulant voluptueusement des hanches est sans aucun doute une séductrice; Oyá qui fait tournoyer ses lourdes jupes et son chasse-mouche brodé de perles fait souffler sur le public, en digne maîtresse du vent, une brise rafraîchissante; Ogún au faciès furieux qui abat une vraie machette d'un côté et de l'autre est identifiable comme guerrier : sans accessoire et esquissé du bout des poignets, comme dans la plupart des cérémonies, le sens du mouvement est beaucoup moins évident.

Les élèves de l'Institut supérieur d'art, qui ont constitué peu à peu un corps de spécialistes érudits, produisent des travaux très standardisés qui s'inspirent des textes de F. Ortiz, des manuels de folklore et de l'enseignement des informateurs-professeurs. Ils comportent des descriptions de danses détaillées geste par geste, des transcriptions de chants et des analyses musicologiques poussées. Si le répertoire palero est riche de nombreuses variantes, il n'en va pas de même du répertoire santero, et a fortiori de celui des batá, et ce sans doute du fait de l'antériorité inattaquable des recherches de F. Ortiz. Quant à rendre compte de la porosité des frontières entre répertoires, il n'en est pas question ici. Une typologie est établie: le rituel qui utilise les batá, appelé güemilere, est défini comme sacré et purement traditionnel, tandis que la présence de tambours bembé est associée à une manifestation festive profane, et celle de violons à une évolution syncrétique contemporaine. Le güemilere modèle est censé comprendre une partie introductive divisée en deux temps : une suite de morceaux instrumentaux jouée uniquement en présence des initiés dans la pièce où reposent les réceptacles à orichas, appelée oru seco ou oru del igbodú, puis une suite chantée jouée devant tout le public. Cet exemple prestigieux d'un rituel bien ordonné suscite l'admiration des religieux, à tel point qu'il arrive aujourd'hui qu'on joue des oru seco lors de rumbas de cajón dédiées aux esprits des morts.

Il est intéressant d'analyser la façon dont sont produites et répétées les catégories des manuels de folklore dans les cours de danse et de musique cubaines. Au groupe «afrocubain » appartiennent les répertoires divisés selon leur appartenance «ethnique »: yoruba, congo, arará... Certains particularismes locaux sont reconnus comme traditionnels, comme la subdivision iyesá, à Matanzas, tandis que les formes " yoruba » orientales sont considérées comme altérées et ne forment pas de sous-genre. Toutefois, nous dit-on, « La musique qui se conserve à Cuba dans les pratiques rituelles afrö̈des est de la musique cubaine », car elle s'est différenciée de sa cousine africaine qui a subi un processus historique différent (León, 1974 : 18). Il en va de même pour le groupe guajiro, malgré ses origines hispaniques. Enfin le groupe proprement «cubain » comprend le son (rural), la rumba (urbaine, profane), le danzón, le chachachá... Le souci de mise en avant de l'authenticité traditionnelle fait place, en dernier ressort, à l'affirmation de la 
cubanité, qualité qui finit même par s'appliquer aux intouchables batá : aujourd'hui, sous l'influence de la musique occidentale, le diamètre de leurs peaux serait en effet différent, et permettrait une meilleure amplitude sonore de l'ensemble (idem : 44), opinion qui rejoint celle d'omo añá cubains réputés (voir Somodeville, cité par Ortiz, 1995 : 51).

Les artistes de l'Ensemble folklorique national aiment à se présenter comme des « améliorateurs de culture ». Tous soulignent le fait qu'ils ont su tirer parti de leur nouveau statut pour se dépasser, notamment en élargissant leurs compétences à tous les répertoires folkloriques. Luis Chacón, omo añá et ex-danseur de la troupe dès 1962, aujourd'hui professeur à l'Institut supérieur d'art de La Havane et à l'école Timba à Rome, explique qu'il a pris des cours de ballet et de danse contemporaine pour améliorer sa technique en rumba. Le célèbre chanteur Lázaro Ross se considérait investi d'une mission, et il s'est employé juste avant sa mort à enregistrer une série exhaustive de disques et à intervenir régulièrement dans les cours pour enfants et les fêtes de quartiers pour «transmettre les racines ", y compris aux Yoruba, son rêve étant de leur faire redécouvrir des chants archaïques dont il pensait être le dépositaire. R. Martínez Furé, ex-directeur artistique de l'Ensemble folklorique, reconnu par ses élèves et ses informateurs comme un érudit en la matière, récolte et revitalise de vieux répertoires inconnus du grand public, tels que les « rumba du temps de l'Espagne », le tambour yuka, les danses du cabildo de Congos reales, le tambour à friction congo loango kinfuiti, les danses et chants arará... Digne successeur de Pablo Roche, il affirme avoir même remis de vieux chants d'orichas au goût du jour. Le fait que la culture livresque réalimente la culture orale est pour lui une source de grande satisfaction. Militant de la reconnaissance des apports africains à la culture cubaine, il compose également des pièces qu'il inclut dans ses spectacles de façon explicitement engagée. De façon générale, les artistes, quelle que soit leur couleur de peau, se sentent extrêmement valorisés et fiers de participer à une telle entreprise.

Les valeurs de résistance, qui font l'unanimité, font majoritairement référence au passé. L'évocation d'une religiosité vivante contemporaine pose quant à elle problème, et ce d'autant plus que ces artistes impliqués sont d'abord et avant tout des religieux. La mise en avant du répertoire yoruba, historiquement légitimé, permet dans une certaine mesure de contourner cette impasse et de présenter des spectacles assez proches de la réalité, ce qui n'est pas le cas du répertoire congo qui subit un traitement différent. Très diversifié, on lui préfère ses avatars obsolètes, danses costumées de cabildos célèbres permettant une mise en scène impressionnante (danse des bâtons, sortie de la Reine...). L'effet est saisissant mais n'a plus grand chose à voir avec les cérémonies paleras et spirites qui ont lieu quotidiennement dans la capitale. Ces dernières présentent en effet un aspect nettement moins séduisant que les danses d'orichas: il n'y a pas de costume pour les possédés, hormis un foulard de couleur noué autour des reins, les participants dansent en masse serrée devant les instruments, et ce ne sont pas des pantomimes : « Leurs danses, y compris chez les possédés, présentent un mélange hétérogène de gestes et de pas, comme s'il s'agissait d'une combinaison d'anciennes danses dont la fonctionnalité représentative aurait été perdue », nous expliquent les manuels (León, 1974 : 63). N'adhérant pas au modèle qui voudrait que toute « danse primitive » implique une corrélation précise entre geste et sens, les danses paleras reçoivent une fois encore les qualificatifs péjoratifs de syncrétique et acculturées.

Il n'existe pas, en effet, de chorégraphie typique dans le palo, et pour cause : le possédé exprime la puissance brute, parfois définie comme sauvage, d'esprits de morts d'origines 
très diverses (Congos, Gitans, Chinois, Espagnols, Indiens, Haïtiens...) qu'il convient d'apprivoiser progressivement, et dont la façon de danser va évoluer et se particulariser. L'assistance n'est pas astreinte à des pas spécifiques, elle improvise à l'aide du vaste vocabulaire chorégraphique cubain, tout en conservant une cohérence rythmique. Les virtuoses dialoguent corporellement avec les percussionnistes et entraînent les autres. Chacun est vivement encouragé à se dépenser au maximum, le but du rituel étant, comme en santería, de faire circuler les énergies bienfaisantes (luz, fuerza, aché) et de provoquer des possessions. Mais au lieu d'exécuter des enchaînements différents selon les morceaux, les participants répètent un même mouvement sur une très longue durée. L'intrusion d'un possédé dans le champ sensoriel ${ }^{32}$ provoquent souvent un lâcher d'émotions : rires, pleurs, tremblements, évanouissements... Toutes sensations non visibles qu'il n'est pas possible de transmettre à un public sous une forme théâtrale classique, privée de sa dimension religieuse.

Les danses d'orichas subissent plus facilement un traitement scénique; elles requièrent par ailleurs un apprentissage minimal, qui laisse souvent une grande partie de l'assistance intimidée et retenue, du moins au début des cérémonies. Mais elles fonctionnent elles aussi sur le principe décrit ci-dessus. Exécutées en " version courte », hors du contexte rituel, il arrivent tout de même qu'elles induisent les transes de danseurs ou de membres du public. De tels événements, lorsqu'ils surviennent, marquent bien toute l'ambiguïté des réalisations de l'Ensemble folklorique national et de la représentation « désacralisée » en général à Cuba (soulignée également par Hagedorn, 2001). Ni le public, ni les acteurs ne sont de véritables athées, et le caractère sacré ou profane d'un événement est défini de façon contingente par les intentions et interactions des acteurs plutôt que par un contexte normé.

La politique castriste à l'égard de la religión a considérablement évoluée ces dix dernières années ${ }^{33}$. Face à une population qui affiche sa croyance et multiplie ses initiations, face à la concurrence avec d'autres lieux de pèlerinage « yoruba » (Miami, Ifé, Salvador de Bahia...) et parallèlement aux alliances stratégiques avec divers mouvements chrétiens, le gouvernement a fini par tolérer les efforts d'institutionnalisation de quelques groupes de religieux qui se développent dans l'île (Argyriadis, Capone, 2004) et publient des manuels, monographies et recueils de mythes. La notion d'identité nationale est repensée de nos jours sous l'angle des «dangers de la globalisation néo-libérale » : un plan de « reconstruction ethnographique » et de revitalisation culturelle a été mis sur pied (Alvarado, 1999 : 19, 21), et le terme folklore abandonné en faveur de celui de culture populaire traditionnelle, qui exclut toutefois le champ de la transmission professionnelle ou médiatique (Esquenazi, 2001 : 7). La promotion du tourisme culturel s'accompagne d'une forte valorisation du thème afro-cubain.

32. Vue de l'extérieur, la scène peut paraître inquiétante : les yeux exorbités, les entités empoignent les personnes de leur choix, les font tourner sur leur dos, les secouent, leur passent vigoureusement les mains sur le corps pour les nettoyer puis leur projettent les bras vers le ciel, leur soufflent diverses substances à la figure, saisissent leur tempes et collent leur front sur leur front en poussant un cri...

33. Dans le milieu des années quatre-vingt, une grande partie de la population, incluant des militants du Parti, a commencé à réclamer plus d'ouverture pour les croyants, ce besoin s'accentuant au fur et à mesure que le pays s'enfonçait dans une crise économique et politique grave. Recherches et publications sur la « religiosité populaire » se sont développées, tandis qu'en 1991 les décisions du IV Congrès du Parti communiste cubain ont donné aux religieux de toutes obédiences le droit de devenir militants. 
Les batá, la rumba et les danses d'orichas fascinent le public de ce tourisme, qui se lance dans l'apprentissage de ce répertoire avec ferveur et évolue souvent vers la pratique religieuse ${ }^{34}$. Sacralisés en quelque sorte une seconde fois par l'aura que leur confère la reconnaissance et l'admiration des universitaires, les batá sont devenus les symboles de la «tradition yoruba », d'une africanité prestigieuse dont seuls les initiés connaîtraient le secret. Le monde de la percussion est aujourd'hui prodigieusement intéressé par ces tambours et se dispute pour savoir si leurs premiers enregistrements au sein de formations de jazz datent de 1975 (Leymarie, 1996 : 16) ou de 1956 (Delannoy, cubafolk.com, 18 juillet 2000). On peut les entendre dans la plupart des orchestres qui se revendiquent comme «afro », par-delà la diversité de leurs styles musicaux (salsa, latin jazz, rap, ragga, rock...).

Comme le souligne M. Augé, « le regard occidental sur les autres n'a cessé d'être méprisant que pour se faire esthétique » (1982 : 11). A Cuba, le mépris, le rejet et le dégoût vont de pair avec la fascination, tant il est vrai que les esclaves et leurs descendants, les «sauvages » intérieurs, gardent prise sur ceux qui les marginalisent grâce à la peur qu'il exercent et aux phantasmes de transgression qu'ils incarnent, à l'instar des « classes dangereuses » (Chevalier, $1984: 25)$ auxquelles ils sont d'ailleurs assimilés. L'esthétisation ouvre une brèche dans ce modèle et permet de nouvelles interactions : ainsi l'afrocubanisme a créé une dynamique légitimante propice à ce que les intéressés eux-mêmes deviennent acteurs des représentations les concernant. A La Havane comme dans la région d'Oyo (et on pourrait en dire autant de Salvador de Bahia, par exemple), la construction de la tradition musicale résulte d'interactions intenses entre informateurs, intellectuels et artistes, les derniers circulant et échangeant leurs théories d'un continent à l'autre. Et c'est bien ce processus qui rapproche et permet la comparaison entre des phénomènes éloignés géographiquement, plutôt que l'hypothèse d'une «tradition ancestrale commune ».

Les artistes-professeurs des stages, festivals et cours «afro-cubains » jouent un rôle de médiateurs de premier plan et contribuent activement et consciemment à la valorisation d'un pan de leurs pratiques auprès du public étranger, notamment européen et nord-américain, tout comme leurs prédécesseurs qui ont su influer par ce biais sur la construction identitaire nationale. Comme dans les années trente, cet intérêt de la part des représentants d'une culture considérée à Cuba comme hautement développée (la culture occidentale) induit à son tour le renforcement de l'estime de soi. Mais cette fois-ci, les artistes-religieux sont les interlocuteurs directs des amateurs d'afro-cubanité, ils vivent de leur art et jouissent déjà d'un statut élevé. Les moins de cinquante ans sont tous passés par l'Ensemble folklorique national, et diplômés des Ecoles d'art ou de l'Institut supérieur d'art. Ils ont des compétences musicales poussées et variées, maîtrisent plusieurs genres folkloriques, différents instruments, d'autres registres musicaux comme le jazz ou la musique classique, ont fait du solfège, de l'harmonie, des cours d'orchestration et de composition et ont lu les textes des ethnologues et musicologues cubains. Ils n'omettent

34. Cuba s'impose comme lieu d'apprentissage privilégié : il n'y a pas de batá au Brésil, et les prix pratiqués aux Etats-Unis ou dans le reste de l'Amérique latine sont prohibitifs. En Afrique l'apprentissage des batá serait impossible pour une personne n'appartenant pas à une famille de musiciens (Leymarie, 1996: 12). A Cuba par contre les stages abondent et les professeurs ne se privent pas pour intégrer leurs élèves à leur lignage rituel. 
cependant jamais de rappeler leur lien avec « la rue », et insistent sur la prépondérance de l'apprentissage par imprégnation, ce qui peut sembler paradoxal.

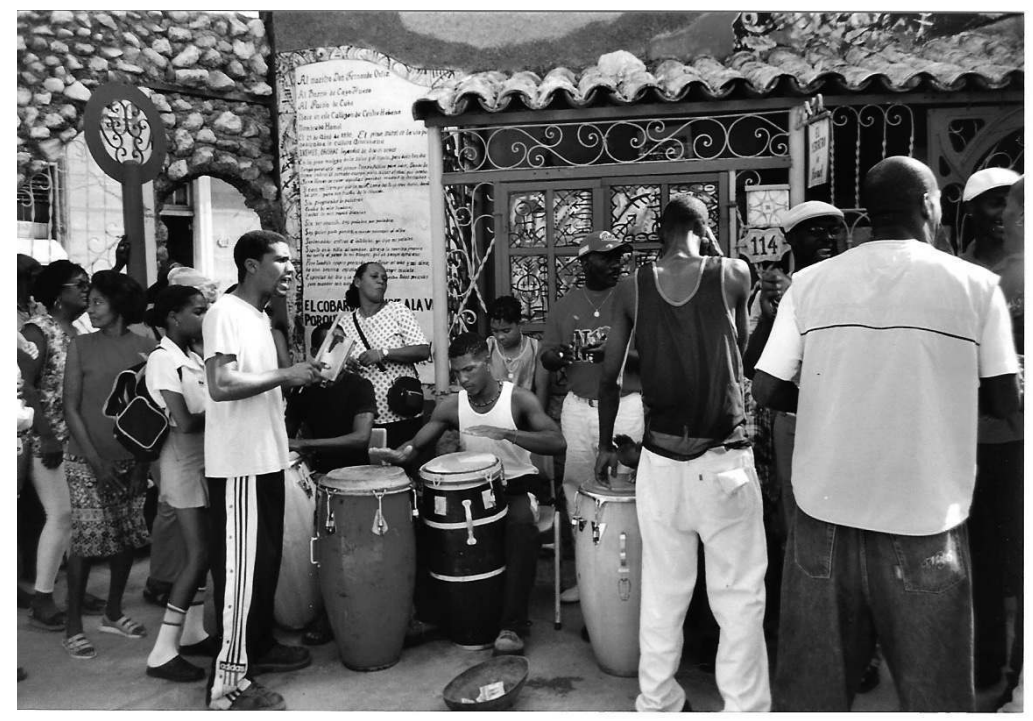

Photo 4 : Rumba jouée sur un orchestre de tumbadoras, dans un des lieux-clé du tourisme culturel à La Havane, le Callejón de Hamel, 2002.

Or il n'en est rien. Leurs performances théâtralisées et codifiées ne sont pas le simple résultat d'une instrumentalisation et leur qualification professionnelle et pédagogique ne les coupe pas du contexte d'exécution quotidien. Ce sont eux, en effet, qu'on retrouve dans les cérémonies et qui se produisent aussi au sein d'orchestres divers ou dans les cabarets. Pour le percussioniste Javier Campos par exemple, qui n'aime pas entendre un orchestre «mal jouer » pendant un rituel, l'implication esthétique exigeante en tant que professeur de l'Institut supérieur d'art est aussi une forme d'engagement, une façon de prouver la valeur de sa croyance. Lázaro Ross expliquait pour sa part en quoi la création de l'Ensemble folklorique national avait marqué selon lui un tournant: "Le peuple s'empara de la scène, cela transforma les choses ». Et c'est bien parce que les discriminations sociales, raciales et religieuses s'expriment encore en termes d'esthétique (gesticulation de singes, laideur, puanteur, grossièreté, saleté, vacarme, sauvagerie) que cette dimension est fortement investie, sur scène comme pendant le rituel. La beauté des danses d'orichas et du phrasé des batá, typologisés, codifiés, " traditionnalisés », hausse la santería au rang de culture. Sur ce point, les aspirations de l'Etat et de la plupart des religieux semblent converger actuellement, et c'est donc autour de cette question que vont s'articuler les luttes de pouvoir et l'évolution de la pratique rituelle à l'intérieur de l'île 35 , concomitante entre autres au développement du tourisme culturel.

35. Reste à faire accepter le statut de religion à part entière, et non de superstition ou de culte local : ce sont plutôt les réseaux transnationaux qui sont investis dans ce but (Argyriadis, Capone, 2004 : 127). 
Les musiciens et danseurs, qui ont fait évoluer un pan de leur répertoire en «l'améliorant» conformément aux critères esthétiques occidentaux dominants et ont contribué en retour à l'évolution de ces mêmes critères sont d'autant plus aptes à toucher un public étranger que ce répertoire, perçu comme totalement exotique, est en réalité le résultat d'un processus qui rapproche bien plus qu'il n'éloigne les parties. Il n'en va pas de même, loin s'en faut, pour les danses et la musique paleras et spirites, exécutées pourtant avec autant de ferveur dans un contexte rituel par ces mêmes artistes mais taxées péjorativement par ces derniers de «plus syncrétiques ». Le répertoire cubain des batá et des danses d'orichas, dont l'efficacité en tant qu'étendard identitaire et culturel valorisant n'est plus à prouver, n'est pas prêt d'être détrôné.

\section{Glossaire des termes vernaculaires utilisés}

Aché, luz, fuerza : l'aché peut se définir comme une force vitale, une énergie présente à des intensités variables dans tous les éléments du cosmos. Ce terme, d'origine santera, est de plus en plus utilisé de façon interchangeable avec les termes force et lumière, ce dernier mot, d'origine spirite, signifiant plus précisément une sorte de force obtenue sans sacrifice sanglant, une connaissance donnée aux morts par les vivants, une énergie que les morts accumulent en aidant les vivants et vice-versa.

Agües (ou abwes) : trio de gros hochets-sonnailles de différentes tailles, utilisé parfois à la place (ou en complément) des batá dans les cérémonies santeras. On les appelle aussi güiros (« donner un guiro en l'honneur de Yemayá ») ou chekerés.

$\boldsymbol{A} \tilde{\boldsymbol{n}} \boldsymbol{a}$ : nom de la force qui vit dans le tambour consacré, qui reçoit régulièrement des offrandes et du sang sacrificiel.

Arará : terme utilisé à Cuba pour désigner les esclaves d'origine ewe-fon et, par extension, la religion de même origine (Regla arará ou foddún).

Babalao : homme initié dans le culte d'ifá, maîtrisant le système divinatoire du même nom. Du yoruba babaláwo (baba ni awo: " père du secret »).

Batá : trio de tambours bimembranophones, ambipercussifs, dont la forme dite " orthodoxe » à Cuba actuellement est celle d'un sablier. Chacune des six membranes est de taille différente et les différents sons obtenus étaient censés reproduire à l'origine les tons de la langue yoruba. Les peaux sont tendues sur une caisse de bois (tronc évidé) avec un cordage de cuir, et accordées avec un emplâtre. Le plus grand des batá est appelé iyá (mère), le moyen itótele et le plus petit okónkolo. L'iyá est également assorti de ceintures de cloches et de grelots autour de chacune de ses membranes.

Bembé : nom générique donné à une fête en l'honneur d'un oricha qui emploie d'autres tambours que les batá (à La Havane aujourd'hui, il s'agit le plus souvent de tumbadoras).

Bongó : paire de petits membranophones cylindriques de taille légèrement différente, produisant des sons aigus, accordés au moyen de clés.

Cabildos : associations qui permettaient aux ressortissants esclaves ou libres de s'entraider et de perpétuer leurs coutumes (avec des restrictions). A la fin du $19^{\mathrm{e}}$ siècle les cabildos « de Noirs » ont été restructurés de force en sociétés d'entraide, dont la plupart ont par la suite périclité suite aux persécutions dont elles étaient l'objet. Un certain nombre a toutefois subsisté de façon informelle, y compris pendant la révolution. 
Cajones : jeu d'idiophones obtenus à l'aide de caisses et de tabourets en bois de différentes tailles, agrémentés parfois de clochettes. Ils sont souvent utilisés, avec les claves, les maracas, les frottoirs, les hochets-sonnailles et les cloches ou fers frappées pour donner des fêtes en l'honneur des morts (cérémonie paleras et spirites) ou tout simplement pour la rumba, avec ou sans complément de tumbadoras.

Cha-cha-chá : genre musical et dansant dérivé du danzón, élaboré par Enrique Jorrín (directeur de l'orchestre América) à la fin des années quarante en s'inspirant du bruit produit sur la piste par les pas glissés des danseurs qui entendaient accentuer ainsi le rythme du güiro.

Chekeré : voir agües.

Claves : nom donné aux deux cylindres en bois entrechoqués qui marquent le rythme dans la rumba et dans le son. C'est aussi le nom des rythmes eux-mêmes : clave de rumba, clave de son.

Comparsas : groupes de danse et de musique se produisant pendant le carnaval, répétant plusieurs mois à l'avance.

Conga : danse de groupe exécutée pendant le carnaval, les pas de base rythmant la marche de la comparsa ou de la foule qui la suit.

Consacré : chargé de force ou d'aché. Se dit de tout réceptacle ayant subi un rituel destiné à cet effet, et qui doit ensuite être régulièrement alimenté en sang sacrificiel.

Danzón : genre musical et dansant dérivé de la contredanse, né à Cuba à la fin du $19^{\mathrm{e}}$ siècle.

Guajiro/a : terme générique désignant les paysans à Cuba. Il désigne également l'ensemble des styles musicaux ruraux décrits comme d'origine ibérique et canarienne, où l'improvisation des paroles prime sur la mélodie en elle-même (décimas, punto guajiro, seguidillas...). Le chant est accompagné de frappement de mains et d'instruments à corde comme la guitare, le tres ou le laúd, et parfois de danses appelées zapateo, où les battements rythmés des pieds des danseurs viennent compléter la mélodie. Au début du $20^{\mathrm{e}}$ siècle, une version idéalisée et romantique de la musique guajira a longtemps été prônée par les défenseurs du blanchissement de la nation (León, 1974 : 95).

Güemilere (ou wemilere) : nom érudit donné à la fête pour les orichas où interviennent les tambours batá. A La Havane on utilise plus couramment le terme tambour (" aller à un tambour pour Changó »).

Güiro : littéralement, calebasse. Ce terme est le plus souvent utilisé pour désigner un frottoir fabriqué avec une calebasse allongée vidée et striée, mais il désigne aussi de gros hochetssonnailles élaborés à partir de grosses calebasses rondes (voir agües).

Ifá : l'oracle, le message. Celui-ci est délivré par des hommes initiés spécialement à ce culte, qui maîtrisent les techniques divinatoires spécifique à ifá : les babalaos.

Iyawó : santero novice, initié depuis moins d'un an.

Lucumí : terme utilisé à Cuba pour désigner les esclaves yoruba et, par extension, la religion d'origine yoruba.

Marímbula : grand lamellophone décrit comme d'origine «bantoue», monté sur une grande caisse de bois (version agrandie des instruments mbila ou sanza).

Náñigos : nom donné aux membres de la société secrète masculine abakuá, originaire selon F. Ortiz du Sud-Est du Nigeria (Calabar).

Nettoyer : Purifier, éliminer les forces néfastes. On procède par contact avec une autre source de force (d'autant plus puissante que le mal est fort), qui incorporera ces dernières, et qu'on jettera en un endroit précis. 
Okónkolo : nom du plus petit des tambours batá.

Olúbatá : possesseur d'un jeu de tambours batá consacrés.

Omo añá : tambourinaires initiés (fils d'Añá) ayant le droit de jouer et de toucher les batá consacrés dans les cérémonies.

Oricha : entité santera, appelée également saint, représentant un ensemble de forces ou de principes. Elle est généralement représentée sous une forme anthropomorphe, possédant plusieurs facettes complémentaires. Les orichas se manifestent par la possession (surtout en dansant) et par différentes techniques divinatoires, comme le lancer de morceaux de noix de coco, le lancer de cauris et les techniques liées à ifá. Outre diverses offrandes, ils ont besoin de fêtes et de sang d'animaux sacrifiés pour être contentés.

Palenque : nom donné à Cuba aux villages d'esclaves fugitifs.

Palo : (palo-monte, mayombe, briyumba, kimbisa...) : culte ordinairement décrit comme d'origine bantoue (entre autres), basé sur le pacte de l'adepte (palero) avec des morts réclamant des offrandes, des fêtes et du sang d'animaux sacrifiés pour travailler et se manifestant par la voyance et la possession. L'usage des herbes et des morceaux de bois (palos) réduits en poudres, considérés comme porteurs de force, y est primordial.

Religión : terme couramment employé à La Havane, désignant l'ensemble des cultes dit «afrocubains ", ainsi qu'une forme locale de spiritisme et la pratique pragmatique du catholicisme. Les adeptes de ces cultes s'appellent eux-mêmes des religieux.

Rumba : genre musical basé sur un rythme spécifique (la clave de rumba). Peut se jouer avec n'importe quels objets suffisamment sonores, l'ensemble formant une polyrythmie complexe. Peut se danser en couple séparé ou sous la forme d'une compétition entre hommes.

Santería (ou Regla Ocha) : culte ordinairement décrit comme d'origine yoruba (entre autres), basé sur l'adoration et l'incorporation d'entités porteuses de grands principes de force appelées orichas ou saints.

Son : genre musical originaire de la région orientale de l'île, qui devint très populaire à La Havane dans les années vingt. La polyrythmie, basée sur la clave de son, était produite à l'origine par une jarre en terre cuite (botija) dans laquelle on soufflait, des lamelles de métal montées sur une caisse de résonance (marímbula), des bâtons entrechoqués (claves), une paire de maracas, une petite paire de tambours (bongó), une calebasse striée frottée (güiro) et une petite guitare à trois paires de cordes (tres). Le chant commence par une succession de couplets puis se poursuit par une semi-improvisation responsoriale où le soliste oriente les refrains du chœur. Le son se danse en couple enlacé, avec des figures simples, les pas suivant le rythme de la clave.

Spiritisme : ce terme désigne, à La Havane, le culte visant à communiquer (par l'intermédiaire de médiums-voyants) avec les défunts, appelés morts de lumière, qui apportent de la lumière aux vivants, et qui prennent de la lumière au contact de ces derniers, l'objectif étant l'évolution des uns et des autres. Ces défunts réclament seulement des offrandes simples et des fêtes, jamais de sang d'animal sacrifié.

Tumbadora : nom donné au tambour appelés conga en Europe.

Tumba francesa : société d'entraide et de divertissement créée par les descendants d'immigrants haïtiens arrivés dans la région orientale de l'île à partir du début du $19^{\mathrm{e}}$ siècle. Par extension, nom donné au répertoire musical et chorégraphique de ces sociétés.

Yuka : trio de hauts tambours cylindriques à une seule membrane clouée sur un tronc évidé, de trois tailles différentes, utilisés dans les zones rurales et décrits comme d'origine «bantoue ». 


\section{Références bibliographiques}

Acosta, Leonardo, 2001. « Interinfluencias y confluencias en la música popular de Cuba y los Estados Unidos », in Rafael Hernandez y John H. Coatsworth (coord.), Culturas encontradas : Cuba y los Estados Unidos, pp. 34-51. La Havane : CIDCC Juan Marinello/University of Harvard (DRCLAS).

Alvarado Ramos, Juan A., 1999. «Introducción », in Cultura popular tradicional cubana (coll.), pp. 9-25. La Havane : CIDCC Juan Marinello/Centro de Antropología.

Angarica, Nicolás Valentín, 1990. " El lucumí al alcance de todos », reproduit in Lázara Menendez, Estudios afrocubanos - selección de lecturas, 3. La Havane : Universidad de La Habana.

ANONYME, 1929. « La represión de la brujería », in Diario de la Marina, 27 de marzo, p. 16. La Havane.

ArgYriadis, Kali,

1998. « Danser avec les entités, danser avec les autres. Le rapport danse-transe-musique dans la religión à La Havane », Percussions, 58, (IX-4), pp. 17-26.

1999. La religión à La Havane. Actualité des représentations et des pratiques cultuelles havanaises. Paris : Editions des Archives Contemporaines.

2000. «Des Noirs sorciers aux babalaos. Analyse du paradoxe du rapport à l'Afrique à La Havane » Cahiers d'Etudes Africaines, 160, pp. 649-674.

2001-2002. "Les Parisiens et la santería: de l'attraction esthétique à l'implication religieuse », Psychopathologie africaine, 1 (XXXI), pp. 17-44.

2005. «El desarrollo del turismo cultural en La Habana y la acusación de mercantilismo », Desacatos, 18. Mexico (mayo-agosto, pp. 29-52).

Argyriadis, Kali et Stefania CAPONE, 2004. «Cubanía et santería. Les enjeux politique de la transnationalisation religieuse (La Havane - Miami) », Civilisations 1-2 ( LI), pp. 81-137.

BAscom, William R., 1952. "Yoruba acculturation in Cuba », in Les afro-américains, Dakar, mémoire de l'IFAN, pp. 163-167.

Bolivar, Natalia \& Natalia del Rio, 2000. Lydia Cabrera en su laguna sagrada, Santiago de Cuba: ed. Oriente.

CAbrera, Lydia, 1993. El Monte. La Havane : ed. Letras Cubanas.

CAPONE, Stefania,

1999. La quête de l'Afrique dans le candomblé. Pouvoir et tradition au Brésil. Paris : Karthala.

2000. «Entre Yoruba et Bantu : l'influence des stéréotypes raciaux dans les études afro-américaines. », Cahiers d'études africaines, 157 (XV), 1, pp. 55-77.

CArPentier, Alejo, 1985. La musique à Cuba. Paris : Gallimard.

Castañeda, Digna, 2001. «Repercusiones de la problemática social de los afronorteamericanos en sectores de la sociedad cubana : vínculos y estratégias comunes (1888-1959) », in Rafael Hernandez y John H. COATSWORTH (coord.), Culturas encontradas: Cuba y los Estados Unidos, pp. 229-241. La Havane : CIDCC Juan Marinello/University of Harvard (DRCLAS).

Castellanos Gonzalez, Israël, 1936. «La brujería y el ñañiguismo desde el punto de vista médico-legal », Revista de técnica policial y penitenciaria, 2-3 (IV), pp. 83-116. La Havane.

CD-Rom collectif, 2000. Atlas etnográfico de Cuba. Cultura popular tradicional (colectivo de investigadores). La Havane : CIDCC Juan Marinello/Centro de Antropología/CEISIC.

Chevalier, Louis, 1984. Classes laborieuses et classes dangereuses. Paris, Hachette. http://www.cubafolk.com (bulletin d'information 1999-2001).ColLECTIF, 1998. Fiestas populares tradicionales cubanas (colectivo de autores). La Havane : ed. Ciencias sociales/CIDCC Juan Marinello. 
Collectif, 1998. Fiestas populares tradicionales cubanas (colectivo de autores). La Havane : ed. Ciencias sociales/CIDCC Juan Marinello.

Cuellar Vizcaino, Manuel,

1948. «Altares de Santa Bárbara », Bohemia, 50, año 40 (12), pp. 57-58 et 90-91. La Havane.

1950. « Un bembé », Bohemia, 37, año 42 (10), pp. 54-58 et 97. La Havane.

DeCORET, Anne, 1998. « Danse sociale et interculturalité : la dansomanie exotique de l'entre-deux-guerres », in Alain Montandon (dir), Sociopoétique de la danse, pp. 505-519. Paris : Anthropos.

Esquenazi Perez, Martha, 2001. Del areito y otros sones. La Havane: ed. Letras cubanas/CIDCC Juan Marinello.

Fernandez Robaina, Tomás,

1994. Hablen paleros y santeros. La Havane : ed. Ciencias Sociales.

Fines, María Antoñica, années 1950. «Libreta de santería », reproduit in Lázara Menendez, 1990, Estudios afrocubanos - selección de lecturas, 3, pp. 17-136. La Havane : Universidad de La Habana.

Garcia Carranza, Araceli, Norma Suarez Suarez \& Alberto Morales, 1996. Cronología Fernando Ortiz. La Havane : Fundación Fernando Ortiz.

Hagedorn, Katherine J., 2001. Divine Utterances. The performance of Afro-Cuban Santeria, Washington \& Londres, Smithsonian Institution Press.

LACHATEÑERÉ, Romulo,

1940. « La influencia Bantú-Yoruba en los cultos afrocubanos ». Estudios Afrocubanos, IV. La Havane.

1952. « Rasgos bantús en la santería », Les Afro-Américains. Dakar : mémoires de l'IFAN.

La Fuente (de), Alejandro, 2001. «Antídotos de Wall Street. Raza y racismo en las relaciones entre Cuba y los Estados Unidos », in Rafael Hernandez y John H. Coatsworth (coord.), Culturas encontradas : Cuba y los Estados Unidos, pp 243-261. La Havane: CIDCC Juan Marinello/University of Harvard (DRCLAS).

LaOYE, I, 1961. «Los tambores yoruba », Actas del folklore, 5, pp. 15-23. 135. La Havane : Centro de Estudios del Folklore del TNC.

LeON, Argeliers,

1961a. «La expresión del pueblo en el Teatro Nacional Cubano », Actas del folklore n 1, La Havane, Centro de Estudios del Folklore del T.N.C., enero, p. 5-7.

1961b. «Creación del Instituto de Etnología y Folklore», Actas del folklore, 10-11-12, pp. 33-35. La Havane : Centro de Estudios del Folklore del TNC.

1971. «Un caso de tradición oral escrita », Islas, 39-40. Las Villas.

1974. Del canto y el tiempo. La Havane : ed. Pueblo y Educación.

Leymarie, Isabelle, 1996. « La migration transatlantique des tambours bàtá », Percussions, 45, pp. 11-19.

MAtory, James Lorand, 2001. «El nuevo imperio yoruba : textos, migración y el auge transatlántico de la nación lucumí, in Rafael Hernandez y John H. Coatsworth (coord.), Culturas encontradas : Cuba y los Estados Unidos, pp. 167-188. La Havane : CIDCC Juan Marinello/University of Harvard (DRCLAS).

Mercier-Balaz Mireille, Daniel Pinos,

1994. Documentaire Asi na 'má, 52 mn. Paris : ATLZA/Planète.

1996. Documentaire Osha Niwé, esclave de la musique, 26 mn. Paris : ATLZA/Planète.

Mestas, María del Cármen, 2000. « Chavalonga, rumbero de ley », Salsa cubana, 13, año 4, pp. 40-41. 


\section{Kali Argyriadis}

ORTIZ, Fernando,

1995 [1906]. Hampa afrocubana: los negros brujos. La Havane : ed. de Ciencias sociales.

1937. « La música sagrada de los negros yorubas en Cuba », Ultra, 13 (III), pp. 77-86. La Havane.

1939. « Brujos o santeros ». Estudios Afrocubanos, 1-4 (III), pp. 85-90. La Havane.

1993 [1950]. La africanía de la música folklórica afrocubana. La Havane : ed. Letras cubanas.

1981 [1951]. Los bailes y el teatro de los negros en el folklore afrocubano. La Havane : ed. Letras Cubanas.

1995 [1954]. Los instrumentos de la música afrocubana. Los tambores batá. La Havane : ed. Letras Cubanas.

PeEL, John D. Y.,

2000. Religious Encounter and the Making of the Yoruba. Bloomington \& Indianapolis: Indiana University Press.

REYes Fortún, José, 2000. « 50 años de rumba en la discografía cubana », Salsa cubana, 11, año 4, pp. 33-37.

Roche Monteagudo, Rafael,

1908a. «Los ñáñigos », in La policía y sus misterios en Cuba, pp. 14-58. La Havane : Imprenta la Prueba.

1908b. «La brujería », in La policía y sus misterios en Cuba, pp. 59-104. La Havane : Imprenta la Prueba.

Rouget, Gilbert, 1965. « Notes et documents pour servir à l'étude de la musique yoruba », Journal de la Société des Africanistes, tome XXXV, pp. 65-107.

Roy, Maya, 1998. Musiques cubaines. Paris : Cité de la musique/Actes Sud.

Torregosa, Jesús, années 1950. «Libreta de santería », reproduit in Lázara MENÉNDEZ, 1990, Estudios afrocubanos - selección de lecturas, 3, pp. 137-268. La Havane : Universidad de La Habana.

Trujillo y Monagas, José, 1882. Los criminales de Cuba. Barcelone : establecimiento tipográfico de Fidel Giró.

URrutía, Gustavo E.,

1935a. «Atarés ! », Diario de la Marina, 10 de noviembre, p. 2. La Havane.

1935b. « Sensemaya », Diario de la Marina, 16 de noviembre, p. 2. La Havane.

1935c. « Hora afrocubana », Diario de la Marina, 18 de diciembre, p. 2. La Havane.

Valero Cossío, Francisco, 1904. «La brujería, el espiritismo y el siglo XX », El nuevo criollo, 13, año I, pp. 2-3, La Havane. 